\title{
ON THE EIGENVALUES OF CERTAIN HERMITIAN OPERATORS
}

\author{
BY \\ HAROLD WIDOM

\section{Chapter I. InTRODUCTION}

In recent years a considerable amount of work has been done on the investigation of the largest eigenvalue of integral equations of the form

$$
\int_{-A}^{A} \rho(x-y) \phi(y) d y=\lambda \phi(x),
$$

where the kernel $\rho(x)$ is real and even, for large values of the parameter $A$.

By a formal use of the Wiener-Hopf technique Carrier [2;3] obtained sharp estimates in the special cases of the kernels

$$
\rho(x)=\int_{|x|}^{\infty} s^{-1} e^{-8} d s,
$$

which arises in the theory of neutron diffusion, and a Hankel function of imaginary argument,

$$
\rho(x)=\frac{1}{2} i H_{0}^{(1)}(i x)=\frac{1}{2 \pi} \int_{-\infty}^{\infty} \frac{e^{-i \xi x}}{\left(1+\xi^{2}\right)^{1 / 2}} d \xi .
$$

In the case of this second kernel Carrier obtained, for the largest eigenvalue of (1.1), the asymptotic expression( $\left.{ }^{1}\right)$

$$
\lambda=1-\frac{\pi^{2}}{8 A^{2}}+\frac{\pi(\pi+2)}{8 A^{3}}+o\left(A^{-3}\right) .
$$

As for general kernels $\rho(x)$, little has been proved but much has been suspected. Bellman and Latter [1] have obtained upper and lower bounds for the largest eigenvalue of (1.1) for quite general $\rho$, these bounds, however, being rather weak for most interesting kernels. More to the point is a conjecture of Kac, Murdock, and Szegö [5]. Assume $\rho(x) \geqq 0$ and $\int_{-\infty}^{\infty}\left(1+x^{2}\right) \rho(x) d x<\infty$. Denote the positive eigenvalues of (1.1) by

Presented to the Society, December 27, 1956, under the title Extreme eigenvalues of Toeplitz matrices; received by the editors January 12, 1957.

(1) Professor Carrier has pointed out that in [3], the 10th line from the bottom of p. 20, the expression $1-\pi / 4(\beta+a)^{2}$ should be replaced by $1-\pi^{2} / 8(\beta+a)^{2}$. 


$$
\lambda_{1, A} \geqq \lambda_{2, A} \geqq \cdots .
$$

Then, this conjecture states,

$$
\lambda_{v, A}=M-\frac{\sigma^{2} \pi^{2} v^{2}}{8 A^{2}}+o\left(A^{-2}\right)
$$

as $A \rightarrow \infty$ for fixed $v$, where $M=\int_{-\infty}^{\infty} \rho(x) d x$ and $\sigma^{2}=\int_{-\infty}^{\infty} x^{2} \rho(x) d x$. (For the basis of this conjecture, and a discussion of related problems, we refer the reader to the excellent introduction of [5].) We shall prove a more precise result here, under slightly different hypotheses. If we denote by $F(\xi)$ the Fourier transform of $\rho(x)$,

$$
F(\xi)=\int_{-\infty}^{\infty} e^{i \xi x} \rho(x) d x
$$

then under certain conditions

$$
\lambda_{v, A}=M-\frac{\sigma^{2} \pi^{2} v^{2}}{8 A^{2}}\left[1+\frac{\alpha}{A}\right]+o\left(A^{-3}\right)
$$

where

$$
\alpha=\frac{1}{\pi} \int_{-\infty}^{\infty}\left\{\frac{F^{\prime}(\xi)}{F(\xi)-M}-\frac{2}{\xi}\right\} \frac{d \xi}{\xi} .
$$

(The methods employed in the proof of this result could be used to further extend the asymptotic expansion of $\lambda_{v, A}$, but further refinements do not seem to be especially interesting.) Note that in this asymptotic expression the first two terms involve only $M=F(0)$ and $\sigma^{2}=-F^{\prime \prime}(0)$, and are local in that they depend only on the behavior of $F(\xi)$ near $\xi=0$, while the next term is clearly nonlocal.

When $F(\xi)=\left(1+\xi^{2}\right)^{-1 / 2}$ the integral involved is easy to evaluate, and we have agreement with Carrier's result, which seems to justify his formal method. (It should be pointed out that the function $\left(1+\xi^{2}\right)^{-1 / 2}$ does not satisfy the hypotheses under which our asymptotic expression is obtained; in fact, we must assume $F(\xi)=O\left(|\xi|^{-2}\right)$ at infinity. This, however, is undoubtedly just a deficiency in the method of proof, and not of the result itself.) The case $\rho(x)=\pi^{-1 / 2} \exp \left(-x^{2}\right)$ is also worth noting. In this case we obtain

$$
\lambda_{1, A}=1-\frac{\pi^{2}}{16 A^{2}}-\frac{\pi^{3 / 2} \zeta(1 / 2)}{16 A^{3}}+o\left(A^{-3}\right) .
$$

This result might be compared with those obtained by Bellman and Latter, loc. cit., for the same kernel.

The discrete analog of (1.1), the Toeplitz matrices, will also be considered in this paper. If 


$$
f(\theta)=\sum_{j=-\infty}^{\infty} c_{j} e^{i j \theta}
$$

is real and even, we define the $n$th Toeplitz matrix of $f$ as

$$
T_{n}=T_{n}(f)=\left(c_{j-k}\right)
$$$$
j, k=0, \cdots, n \text {. }
$$

Denote the eigenvalues of $T_{n}$ by

$$
\lambda_{1, n} \geqq \lambda_{2, n} \geqq \cdots \geqq \lambda_{n+1, n} .
$$

It is proved in [5, Chapter III], that if $f(\theta)$ achieves its maximum at $\theta=0$ (and at that point only) and is sufficiently regular, then

$$
\lambda_{v, n}=M-\frac{\sigma^{2} \pi^{2} v^{2}}{2 n^{2}}+o\left(n^{-2}\right)
$$

for fixed $v$ as $n \rightarrow \infty$, where $M=f(0)$ and $\sigma^{2}=-f^{\prime \prime}(0)$. This is the exact analog of the first two terms of the expansion (1.2). We shall prove the analog of all of (1.2), namely

where

$$
\lambda_{v, n}=M-\frac{\sigma^{2} \pi^{2} v^{2}}{2(n+1)^{2}}\left[1+\frac{\alpha}{n+1}\right]+o\left(n^{-3}\right)
$$

$$
\alpha=\frac{1}{\pi} \int_{-\pi}^{\pi}\left\{\frac{f^{\prime}(\theta)}{f(\theta)-M}-2 \csc \theta\right\} \cot \frac{1}{2} \theta d \theta .
$$

The author wishes to express his appreciation to Professor Mark Kac, who introduced the author to the problems considered here, and whose assistance during their solution was invaluable.

\section{Chapter II. Toeplitz matrices}

2.1. We shall be concerned with the class of functions $f(\theta)$ satisfying the following

Condition A. Let $f(\theta)$ be continuous and periodic with period $2 \pi$. Let $\max f(\theta)=f(0)=M$ and let $\theta=0$ be the only value of $\theta(\bmod 2 \pi)$ for which this maximum is reached. Moreover, we assume that $f(\theta)$ is even, and has continuous derivatives up to the fourth order in some neighborhood of $\theta=0$. Finally, let $\sigma^{2}=-f^{\prime \prime}(0) \neq 0$.

The main result of the chapter is

TheOREM 2.1. Let $f(\theta)$ satisfy Condition $\mathrm{A}$, and denote the eigenvalues of the matrix $T_{n}$ by $\lambda_{v, n}[c f .(1.3)-(1.4)]$. Then as $n \rightarrow \infty$ we have, for fixed, $v$ $=1,2, \cdots$,

$$
\lambda_{v, n}=M-\frac{\sigma^{2} \pi^{2} v^{2}}{2(n+1)^{2}}\left[1+\frac{\alpha}{n+1}\right]+o\left(n^{-3}\right),
$$


where

$$
\alpha=\alpha(f)=\frac{1}{2 \pi} \int_{-\pi}^{\pi} \csc ^{2} \frac{1}{2} \theta \log \left[\frac{M-f(\theta)}{2 \sigma^{2}} \cot ^{2} \frac{1}{2} \theta\right] d \theta .
$$

The assumption that the maximum of $f(\theta)$ is achieved at $\theta=0$ is unimportant; the point 0 may be replaced by any $\theta_{0}$. Since the Toeplitz matrix corresponding to any translate of $f(\theta)$ has exactly the same eigenvalues as that corresponding to $f(0)$ itself, it is easy to modify the theorem so that it will hold in the more general case.

2.2. We shall first prove the main theorem for the case when $f(\theta)$ is a trigonometric polynomial and satisfies the additional

Condition $\mathrm{A}_{1}$. All the complex zeros of $f(\theta)-M$ are simple.

The result for general functions will then follow by fairly simple approximation arguments.

Let

$$
f(\theta)=\sum_{j=-k}^{k} c_{j} e^{i j \theta},
$$$$
c_{k} \neq 0
$$

where, since $f(\theta)$ is even, $c_{-j}=c_{j}(j=1, \cdots, k)$. Denote by $T$ the infinite matrix

$$
\left(c_{p, q}\right)=\left(c_{p-q}\right), \quad \quad p, q=0,1, \cdots .
$$

Then if $E_{n}$ is the matrix which takes the vector $\left\{x_{0}, \cdots, x_{n}, x_{n+1}, \cdots\right\}$ into $\left\{x_{0}, \cdots, x_{n}, 0, \cdots\right\}$ we have $T_{n}=E_{n} T E_{n}$. The condition that $\left\{x_{0}, \cdots, x_{n}\right\}$ be an eigenvector of $T_{n}$ corresponding to the eigenvalue $\lambda$ is that

$$
E_{n} T X=\lambda X
$$

where $X$ is the infinite vector $\left\{x_{0}, \cdots, x_{n}, 0, \cdots\right\}$.

For any vector $Y=\left\{y_{0}, \cdots, y_{n}, y_{n+1}, \cdots\right\}$ with $\sum\left|y_{j}\right|^{2}<\infty$ we introduce the generating function

$$
F_{Y}(z)=\sum_{j=0}^{\infty} y_{j} z^{j}
$$

regular inside the unit circle. We can then write (2.3) in the equivalent form

$$
\frac{d^{r}}{d z^{r}}\left[F_{T X}(z)-\lambda F_{X}(z)\right]_{z=0}=0, \quad 0 \leqq r \leqq n,
$$

which, for convenience we split into the two conditions

$$
\frac{d^{r}}{d z^{r}}\left[F_{T X}(z)-\lambda F_{X}(z)\right]_{z=0}=0, \quad 0 \leqq r \leqq n-k,
$$




$$
\frac{d^{r}}{d z^{r}}\left[F_{T X}(z)-\lambda F_{X}(z)\right]_{z=0}=0, \quad n-k<r \leqq n .
$$

Now (2.4) is equivalent to $E_{n-k} T X=\lambda E_{n-k} X$ which, when written out, becomes the system of equations

$$
\begin{array}{lr}
\sum_{p=0}^{r+k} c_{r-p} x_{p}=\lambda x_{r}, & 0 \leqq r \leqq k, \\
\sum_{p=r-k}^{r+k} c_{r-p} x_{p}=\lambda x_{r}, & k<r \leqq n-k,
\end{array}
$$

or

$$
\begin{aligned}
& x_{r+k}=c_{k}^{-1}\left[\left(\lambda-c_{0}\right) x_{r}-\sum_{p=\max (0, r-k)}^{r-1} c_{r-p} x_{p}-\sum_{p=r+1}^{r+k-1} c_{r-p} x_{p}\right] \\
& 0 \leqq r \leqq n-k .
\end{aligned}
$$

As for (2.4), we calculate $F_{T X}(z)$, obtaining

$$
\begin{aligned}
F_{T X}(z)= & c_{0} F_{X}(z)+c_{1} z F_{X}(z)+\cdots+c_{k} z^{k} F_{X}(z) \\
& +c_{1} z^{-1}\left[F_{X}(z)-x_{0}\right]+c_{2} z^{-2}\left[F_{X}(z)-x_{0}-x_{1} z\right]+\cdots \\
& +c_{k} z^{-k}\left[F_{X}(z)-x_{0}-x_{1} z-\cdots-x_{k-1} z^{k}\right] .
\end{aligned}
$$

Thus if we introduce the polynomial

(2.7) $P(z)=P(z ; \lambda)=c_{k} z^{2 k}+\cdots+c_{1} z^{k+1}+\left(c_{0}-\lambda\right) z^{k}+c_{1} z^{k-1}+\cdots+c_{k}$ we have

$$
z^{k}\left[F_{T X}(z)-\lambda F_{X}(z)\right]=P(z) F_{X}(z)-\sum_{q=0}^{k-1} A_{q} z^{q}
$$

where we have set

$$
A_{q}=\sum_{p=0}^{q} c_{p-q+k} x_{p}, \quad 0 \leqq q \leqq k-1 .
$$

Now by (2.8), we see that (2.5) is equivalent to

$$
\frac{d^{r}}{d z^{r}}\left[P(z) F_{X}(z)-\sum_{q=0}^{k-1} A_{q} z^{q}\right]_{z=0}=0, \quad n<r \leqq n+k .
$$

Since (2.4) is equivalent to

$$
\frac{d^{r}}{d z^{r}}\left[P(z) F_{X}(z)-\sum_{q=0}^{k-1} A_{q} z^{q}\right]_{z=0}=0, \quad 0 \leqq r \leqq n,
$$


we see, noting that $P(0) \neq 0$, that (2.4) and (2.5) together are equivalent to (2.5) and

$$
\frac{d^{r}}{d z^{r}}\left[F_{X}(z)-P(z)^{-1} \sum_{q=0}^{k-1} A_{q} z^{q}\right]_{z=0}=0, \quad n<r \leqq n+k .
$$

But since $F_{X}^{(n+1)}(0)=\cdots=F_{X}^{(n+k)}(0)=0,(2.10)$ is equivalent to

$$
\frac{d^{n+p+1}}{d z^{n+p+1}}\left[P(z)^{-1} \sum_{q=0}^{k-1} A_{q} z^{q}\right]_{z=0}=0, \quad 0 \leqq p \leqq k-1 .
$$

Recapitulating, we have shown that $\left\{x_{0}, \cdots, x_{n}\right\}$ is an eigenvector of $T_{n}$ corresponding to the eigenvalue $\lambda$ if and only if we have simultaneously (2.6) and (2.11), where the $A_{q}$ in (2.11) are defined by (2.9). Note that (2.11) involves only the components $x_{0}, \cdots, x_{k-1}$, so that if $x_{0}, \cdots, x_{k-1}$ satisfy (2.11) we can define $x_{k}, \cdots, x_{n}$ successively by means of (2.6) to obtain an eigenvector $\left\{x_{0}, \cdots, x_{n}\right\}$. Thus the multiplicity of $\lambda$ is exactly the number of linearly independent solutions for $\left\{x_{0}, \cdots, x_{k-1}\right\}$ of (2.11) and (2.9). Now (2.9) gives an isomorphism between the set of all $\left\{A_{0}, \cdots, A_{k-1}\right\}$ and all $\left\{x_{0}, \cdots, x_{k-1}\right\}$. It follows that the multiplicity of $\lambda$ is the number of linearly independent solutions of (2.11) for the quantities $A_{0}, \cdots, A_{k-1}$.

In order to bring (2.11) to a workable form we must consider the zeros of $P(z)$. Since, by (2.7) and (2.2),

$$
P\left(e^{i \theta} ; \lambda\right)=e^{i k \theta}[f(\theta)-\lambda],
$$

$P(z)$ will have double zeros only if $f(\theta)-\lambda$ does. Now $f^{\prime}(\theta)$ has at most $2 k$ zeros $(\bmod 2 \pi)$, say $\theta_{1}^{\prime}, \cdots, \theta_{j}^{\prime}(j \leqq 2 k)$. Then if $\lambda_{i}=f\left(\theta_{i}^{\prime}\right), f(\theta)-\lambda$ will have only simple zeros as long as $\lambda \neq \lambda_{i}(i=1, \cdots, j)$. Thus if we choose $\delta>0$ so small that no $\lambda_{i}$ is contained in the open interval $(M, M-\delta), P(z)$ will have only simple zero for $\lambda \in(M, M-\delta)$.

That we may exclude the possibility that $\lambda=M$ is easy. For if $\left\{x_{0}, \cdots, x_{n}\right\}$ is an eigenvector with $\sum\left|x_{j}\right|^{2}=1$ corresponding to the eigenvalue $\lambda$, and we set $\phi(\theta)=\sum x_{j} e^{i j \theta}$, we have

Since

$$
\lambda=\sum_{p, q=0}^{n} c_{p-q} x_{p} \bar{x}_{q}=\frac{1}{2 \pi} \int_{-\pi}^{\pi} f(\theta)|\phi(\theta)|^{2} d \theta .
$$

$$
\frac{1}{2 \pi} \int_{-\pi}^{\pi}|\phi(\theta)|^{2} d \theta=1
$$

and $f(\theta)=M$ at a single point only, it follows that $\lambda<M$.

Let $\rho$ run over the various zeros of $P(z)$. Then we have

$$
\frac{1}{P(z)}=\sum_{\rho} \frac{1}{P^{\prime}(\rho)(z-\rho)}
$$


and (2.11) can be written

$$
\sum_{q=0}^{k-1}\left(\sum_{\rho} \frac{1}{P^{\prime}(\rho) \rho^{n+p-q+2}}\right) A_{q}=0, \quad 0 \leqq p \leqq k-1 .
$$

Now we see from (2.7) that the zeros of $P(z)$ occur in reciprocal pairs, so we may replace each $\rho$ in (2.13) by $1 / \rho$. Since $P\left(z^{-1}\right)=z^{2 k} P(z)$, we see that (2.13) becomes

$$
\sum_{q=0}^{k-1}\left(\sum_{\rho} \frac{\rho^{2 k+n+p-q}}{P^{\prime}(\rho)}\right) A_{q}=0, \quad 0 \leqq p \leqq k-1 .
$$

Hence we have shown that for $\lambda$ in a sufficiently small open interval $(M, M-\delta)$, the multiplicity of $\lambda$ is $k$ minus the rank of the matrix

$$
\left(\sum_{\rho} \frac{\rho^{2 k+n+p-q}}{P^{\prime}(\rho)}\right), \quad \quad p, q=1, \cdots, k .
$$

(Our substitution of $1, \cdots, k$ for $0, \cdots, k-1$ as the range of $p$ and $q$ is clearly irrelevant since each entry of the matrix involves only $p-q$.)

2.3. Denote by $D$ the determinant of the matrix (2.14). Then we have

$$
D=\sum_{\tau} \operatorname{sgn} \tau\left(\sum_{\rho_{1}} \frac{\rho_{1}^{2 k+n+1-\tau(1)}}{P^{\prime}\left(\rho_{1}\right)}\right) \cdots\left(\sum_{\rho_{k}} \frac{\rho_{k}^{2 k+n+k-\tau(k)}}{P^{\prime}\left(\rho_{k}\right)}\right)
$$

where $\tau$ runs over all permutations of $1, \cdots, k$ and each $\rho_{i}$ runs over all the zeros $\rho$ of $P(z)$. Changing the order of the summations, we have

$$
D=\sum_{\rho_{1}, \cdots, \rho_{k}} \frac{\left(\rho_{1} \cdots \rho_{k}\right)^{2 k+n}}{P^{\prime}\left(\rho_{1}\right) \cdots P^{\prime}\left(\rho_{k}\right)} \sum_{\tau}(\operatorname{sgn} \tau) \rho_{1}^{1-\tau(1)} \cdots \rho_{k}^{k-\tau(k)} .
$$

Now

$$
\sum_{\tau}(\operatorname{sgn} \tau) \rho_{1}^{1-\tau(1)} \cdots \rho_{k}^{k-\tau(k)}
$$

is simply a Vandermonde determinant, whose value is

$$
\rho_{1}^{1-k} \rho_{2}^{2-k} \cdots \rho_{k-1}^{-1} \prod_{s<t}\left(\rho_{s}-\rho_{t}\right)
$$

so

$$
D=\sum_{\rho_{1}, \cdots, \rho_{k}} \frac{\left(\rho_{1} \cdots \rho_{k}\right)^{k+n+1}}{P^{\prime}\left(\rho_{1}\right) \cdots P^{\prime}\left(\rho_{k}\right)} \rho_{2} \rho_{3} \cdots \rho_{k}^{k-1} \prod_{s<t}\left(\rho_{s}-\rho_{t}\right) .
$$

Since the factor $\Pi\left(\rho_{s}-\rho_{t}\right)$ in (2.15) will vanish if any two of the $\rho_{i}$ are equal, we may assume the summation is taken only over distinct sets of zeros $\rho_{1}, \cdots, \rho_{k}$. 
Take any set $S$ of $k$ distinct $\rho$ 's, say $S=\left\{\tilde{\rho}_{1}, \cdots, \tilde{\rho}_{k}\right\}$, and let $\sum s$ be that part of the sum for which each $\rho_{i} \in S$. Then if $\tau$ is a permutation of the integers $1, \cdots, k$ the typical term of $\sum_{S}$ is

$$
\begin{aligned}
\frac{\left(\tilde{\rho}_{\tau(1)} \cdots \tilde{\rho}_{\tau(k)}\right)^{k+n+1}}{P^{\prime}\left(\rho_{\tau(1)}\right) \cdots} P^{\prime}\left(\rho_{\tau(k)}\right) & \tilde{\rho}_{\tau(2)}^{2} \tilde{\rho}_{\tau(3)} \cdots \tilde{\rho}_{\tau(k)}^{k-1} \prod_{s<t}\left(\tilde{\rho}_{\tau(s)}-\tilde{\rho}_{\tau(t)}\right) \\
= & {\left[\prod_{\tilde{\rho} \in S} \frac{\tilde{\rho}^{k+n+1}}{P^{\prime}(\tilde{\rho})}\right]\left[\prod_{s<t}\left(\tilde{\rho}_{s}-\tilde{\rho}_{t}\right)\right](\operatorname{sgn} \tau) \tilde{\rho}_{\tau(2)} \tilde{\tilde{\rho}}_{\tau(3)}^{2} \cdots \tilde{\rho}_{\tau(k)}^{k-1} . }
\end{aligned}
$$

Again,

$$
\sum_{\tau}(\operatorname{sgn} \tau) \tilde{\rho}_{\tau(2)} \tilde{\rho}_{r(3)}^{2} \cdots \tilde{\rho}_{\tau(k)}^{k-1}
$$

is a Vandermonde determinant whose value is

$$
\prod_{s<t}\left(\tilde{\rho}_{s}-\tilde{\rho}_{t}\right) .
$$

Therefore, summing (2.16) over $\tau$, we have

$$
\sum_{S}=\left[\prod_{\rho \in S} \frac{\tilde{\rho}^{k+n+1}}{P^{\prime}(\tilde{\rho})}\right]\left[\prod_{s<t}\left(\tilde{\rho}_{s}-\tilde{\rho}_{t}\right)^{2}\right] .
$$

Finally, summing over all choices of $S$, we obtain

$$
D=\sum_{\tilde{\rho}_{1}, \cdots, \tilde{\rho}_{k}} \frac{\left(\tilde{\rho}_{1} \cdots \tilde{\rho}_{k}\right)^{k+n+1}}{P^{\prime}\left(\tilde{\rho}_{1}\right) \cdots P^{\prime}\left(\tilde{\rho}_{k}\right)} \operatorname{II}_{s<t}\left(\tilde{\rho}_{s}-\tilde{\rho}_{t}\right)^{2},
$$

where here the summation extends over all combinations of $k$ of the zeros $\rho$.

2.4. We shall presently show that of the $C_{2 k, k}$ terms in the sum (2.17), all but two can be neglected. Before we do this, however, we have to examine more carefully the distribution of the $\rho$ 's.

The function $f(\theta)-M$ has, by Condition $\mathrm{A}$, the double zero $\theta=0$ and no other real zeros $(\bmod 2 \pi)$. Moreover, by Condition $A$, it has $2 k-2$ distinct complex zeros $(\bmod 2 \pi)$

$$
\theta_{ \pm 2}^{*}, \cdots, \theta_{ \pm k}^{*}
$$

where $\theta_{2}^{*}, \cdots, \theta_{k}^{*}$ are the zeros in the lower half-plane, $\theta_{-2}^{*}, \cdots, \theta_{-k}^{*}$ those in the upper half-plane.

If $\lambda<M$ but sufficiently close to $M, f(\theta)-\lambda$ will have zeros

$$
\theta_{ \pm 1}, \theta_{ \pm 2}, \cdots, \theta_{ \pm k}
$$

where $\theta_{j} \rightarrow \theta_{j}^{*}$ as $\lambda \rightarrow M(j= \pm 2, \cdots, \pm k), \theta_{1}$ is the positive real zero of $f(\theta)-\lambda$, and $\theta_{-1}=-\theta_{1}$.

Correspondingly [cf. (2.12)] $P(z ; M)$ has a double zero at $z=1$, and, off the unit circle, the $2 k-2$ distinct zeros 


$$
\stackrel{*}{\rho_{ \pm 2}}, \cdots, \stackrel{*}{\rho_{ \pm k}}, \quad \stackrel{\rho_{j}^{*}}{*}=e^{i \theta_{j}^{*}} \quad(j= \pm 2, \cdots, \pm k)
$$

where $\rho_{2}^{*}, \cdots, \rho_{k}^{*}$ are of absolute value greater than 1 , and $\rho_{-2}^{*}, \cdots, \rho_{-k}^{*}$ of absolute value less than 1 .

If $\lambda<M$ but sufficiently close to $M, P(z ; \lambda)$ will have zeros

$$
e^{ \pm i \theta_{1}}, \quad \rho_{ \pm 2}, \cdots, \rho_{ \pm k}, \quad \rho_{j}=e^{i \theta_{j}} \quad(j= \pm 2, \cdots, \pm k)
$$

where $\rho_{j} \rightarrow \rho_{j}^{*}$ as $\lambda \rightarrow M(j= \pm 2, \cdots, \pm k)$.

Define

$$
D_{1}=f^{\prime}\left(\theta_{1}\right) \frac{P^{\prime}\left(\rho_{2}\right) \cdots P^{\prime}\left(\rho_{k}\right)}{\left(\rho_{2} \cdots \rho_{k}\right)^{k+n+1}} \prod_{s<t ; s, t=2, \cdots, k}\left(\rho_{s}-\rho_{t}\right)^{-2} D .
$$

Then $D_{1}$ is a sum of terms all but two of which, as we shall show, vanish exponentially as $n \rightarrow \infty$. Note that since $\min _{2 \leqq j \leqslant k}\left|\rho_{j}^{*}\right|>1$ we can find a constant $\mu>1$ so that

$$
\left|\rho_{j}\right| \geqq \mu, \quad\left|\rho_{-j}\right| \leqq 1 / \mu \quad j=2, \cdots, k,
$$

for $\lambda$ sufficiently close to $M$.

Consider a term in the expansion of $D_{1}$ by means of $(2.17)$ in which either

(a) not all the zeros $\rho_{2}, \cdots, \rho_{k}$ are among the $\tilde{\rho}_{i}$, or

(b) at least one of the zeros $\rho_{-2}, \cdots, \rho_{-k}$ is among the $\tilde{\rho}_{i}$.

The factor

$$
P^{\prime}\left(\rho_{2}\right) \cdots P^{\prime}\left(\rho_{k}\right) \prod_{s<t ; s, t=2, \cdots, k}\left(\rho_{s}-\rho_{t}\right)^{-2}
$$

occurring in (2.18) is clearly bounded. We have, therefore, to investigate the behavior of

$$
f^{\prime}\left(\theta_{1}\right)\left(\frac{\tilde{\rho}_{1} \cdots \tilde{\rho}_{k}}{\rho_{2} \cdots \rho_{k}}\right)^{k+n+1} \frac{\operatorname{II}_{s<t}\left(\tilde{\rho}_{s}-\tilde{\rho}_{t}\right)^{2}}{P^{\prime}\left(\tilde{\rho}_{1}\right) \cdots P^{\prime}\left(\tilde{\rho}_{k}\right)} .
$$

Under either of the conditions (a) or (b) above we have, by (2.19),

$$
\left|\left(\frac{\tilde{\rho}_{1} \cdots \tilde{\rho}_{k}}{\rho_{2} \cdots \rho_{k}}\right)^{k+n+1}\right| \leqq \mu^{-(k+n+1)}<\mu^{-n}
$$

The rest of (2.20), namely

$$
f^{\prime}\left(\theta_{1}\right) \frac{\prod_{s<t}\left(\tilde{\rho}_{s}-\tilde{\rho}_{t}\right)^{2}}{P^{\prime}\left(\tilde{\rho}_{1}\right) \cdots P^{\prime}\left(\tilde{\rho}_{k}\right)},
$$

we shall show is bounded. Since each $P^{\prime}\left(\rho_{j}^{*}\right) \neq 0, P^{\prime}\left(\rho_{i}\right)$ will be bounded away from 0 unless $\rho_{i}=e^{ \pm i \theta_{1}}$. We consider three cases.

( $\alpha$ ) Neither $e^{ \pm i \theta_{1}}$ is among the $\tilde{\rho}_{i}$. Then (2.21) is bounded. 
( $\beta$ ) Either $e^{i \theta_{1}}$ or $e^{-i \theta_{1}}$ is among the $\tilde{\rho}_{i}$, but not both. In this case (2.21) is [cf. (2.12)]

$$
O\left(\left|\frac{f^{\prime}\left(\theta_{1}\right)}{P^{\prime}\left(e^{ \pm i \theta_{1}}\right)}\right|\right)=O(1) .
$$

$(\gamma)$ Both $e^{ \pm i \theta_{1}}$ are among the $\tilde{\rho}_{i}$. Then

$$
\frac{f^{\prime}\left(\theta_{1}\right)}{P^{\prime}\left(\rho_{1}\right) \cdots P^{\prime}\left(\rho_{k}\right)}=O\left(\frac{\left|f^{\prime}\left(\theta_{1}\right)\right|}{\left|P^{\prime}\left(e^{i \theta_{1}}\right)\right|^{2}}\right)=O\left(\left|f^{\prime}\left(\theta_{1}\right)\right|^{-1}\right)=O\left(\theta_{1}^{-2}\right),
$$

since $f^{\prime \prime}(0) \neq 0$. But

$$
\prod_{s<t}\left(\tilde{\rho}_{s}-\tilde{\rho}_{t}\right)^{2}=O\left(\left|e^{i \theta_{1}}-e^{-i \theta_{1}}\right|^{2}\right)=O\left(\theta_{1}^{2}\right) .
$$

Thus (2.21) is $O(1)$.

Collecting our results, we have shown that in the expansion of $D_{1}$ by means of (2.17), the sum of those terms for which either (a) or (b) above hold is at most $O\left(\mu^{-n}\right)$ as $n \rightarrow \infty$. There are just two remaining terms, namely that for which the $\tilde{\rho}_{i}$ are $e^{i \theta_{1}}, \rho_{2}, \cdots, \rho_{k}$ and that for which the $\tilde{\rho}_{i}$ are $e^{-i \theta_{1}}$, $\rho_{2}, \cdots, \rho_{k}$. Keeping in mind that

$$
i P^{\prime}\left(e^{ \pm i \theta_{1}}\right)= \pm e^{ \pm i(k-1) \theta_{1}} f^{\prime}\left(\theta_{1}\right)
$$

we have, therefore,

$$
D_{1}=i\left[e^{i(n+2) \theta_{1}} \prod_{s=2}^{k}\left(e^{i \theta_{1}}-\rho_{s}\right)^{2}-e^{-i(n+2) \theta_{1}} \prod_{s=2}^{k}\left(e^{-i \theta_{1}}-\rho_{s}\right)^{2}\right]+O\left(\mu^{-n}\right) .
$$

2.5. In calculating the zeros of $D_{1}$ we shall have to make use of the fact that the error term $O\left(\mu^{-n}\right)$ is negligible. However, it is clear that if $\theta_{1}$ is of the order of $\mu^{-n}$, then the "dominant" term in (2.22) is no larger than the error term. To eliminate this difficulty we shall show that we may assume $\theta_{1}$ is not too close to 0 , or equivalently that $\lambda=f\left(\theta_{1}\right)$ is not too close to $M$.

Lemma. There exists a constant $A>0$ such that if $\lambda$ is an eigenvalue of $T_{n}$, with $n$ sufficiently large, we have $\lambda \leqq M-A n^{-2}$.

The proof is simply a refinement of the proof given in $\$ 2.2$ that $\lambda \neq M$. Let $\left\{x_{0}, \cdots, x_{n}\right\}$ be an eigenvector corresponding to $\lambda, \sum\left|x_{j}\right|^{2}=1$. Letting $\phi(\theta)=\sum x_{j} e^{i j \theta}$ we have, as before,

$$
\lambda=\frac{1}{2 \pi} \int_{-\pi}^{\pi} f(\theta)|\phi(\theta)|^{2} d \theta .
$$

For sufficiently small $\theta$, we have

$$
f(\theta) \leqq M-\frac{1}{4} \sigma^{2} \theta^{2} \quad\left[\sigma^{2}=-f^{\prime \prime}(0)\right],
$$


and, by Condition A,

$$
\max _{|\theta| \geqq n^{-1}} f(\theta)=f\left(n^{-1}\right) \leqq M-\frac{1}{4} \sigma^{2} n^{-2}
$$

for sufficiently large $n$. Thus

$$
\begin{aligned}
\lambda \leqq \frac{1}{2 \pi} \int_{-n^{-1}}^{n^{-1}}(M- & \left.\frac{1}{4} \sigma^{2} \theta^{2}\right)|\phi(\theta)|^{2} d \theta+\frac{1}{2 \pi}\left(M-\frac{1}{4} \sigma^{2} n^{-2}\right) \int_{|\theta| \geq n^{-1}}|\phi(\theta)|^{2} d \theta \\
& =M-\frac{\sigma^{2}}{8 \pi}\left(\int_{-n^{-1}}^{n^{-1}} \theta^{2}|\phi(\theta)|^{2}+n^{-2} \int_{|\theta| \geq n^{-1}}|\phi(\theta)|^{2} d \theta\right) \\
& =M-\frac{\sigma^{2}}{8 \pi}\left(2 \pi n^{-2}-\int_{-n^{-1}}^{n^{-1}}\left(n^{-2}-\theta^{2}\right)|\phi(\theta)|^{2} d \theta\right)
\end{aligned}
$$

Since, by Schwarz's inequality, $|\phi(\theta)|^{2} \leqq n+1<3 n$ for all $\theta$,

$$
\int_{-n^{-1}}^{n^{-1}}\left(n^{-2}-\theta^{2}\right)|\phi(\theta)|^{2} d \theta \leqq 4 n^{-2},
$$

so (2.23) gives

$$
\lambda \leqq M-\frac{\sigma^{2}}{4 \pi}(\pi-2) n^{-2}
$$

2.6. It is convenient now to consider $D$ and $D_{1}$ functions of the complex variable $\theta_{1}$. For $\theta_{1}$ sufficiently close to $0, \lambda=f\left(\theta_{1}\right)$ is close to $M$, and the zeros $\rho_{ \pm 2}, \cdots, \rho_{ \pm k}$, being analytic functions of $\lambda\left[\rho_{ \pm 2}^{*}, \cdots, \rho_{ \pm k}^{*}\right.$ are simple zeros of $P(z ; M)$ ], are also analytic functions of $\theta_{1}$. The remaining zeros $e^{ \pm i \theta_{1}}$ are certainly analytic. Thus $D$, the determinant of the matrix (2.14), is analytic in a region of the form $0<\left|\theta_{1}\right|<\delta$. Consequently $D_{1}$ is analytic in this same region. (Actually it follows from (2.22) that $D_{1}$ is bounded near $\theta_{1}=0$ so it is regular in the entire disc $\left|\theta_{1}\right|<\delta$.)

We know from the lemma of $\$ 2.5$ that $D_{1}$ is free of zeros in some region $\left|\theta_{1}\right| \leqq B n^{-1}$. We shall confine our attention to the ring

$$
\frac{B}{n}<\left|\theta_{1}\right|<\left(N+\frac{1}{2}\right) \frac{\pi}{n}
$$

where $N$ is arbitrarily large but fixed. We may clearly assume, and we do, that $B<\pi$. It will be shown that $D_{1}\left(\theta_{1}\right)$ has exactly $2 N$ zeros in the region (2.24), these being simple.

Define the polynomial 


$$
Q(z)=\prod_{s=2}^{k}\left(z-\dot{\rho_{s}}\right)
$$

Then the expressions $\Pi\left(e^{ \pm i \theta_{1}}-\rho_{s}\right)^{2}$ occurring in (2.22) will both be quite close to $Q(1)^{2}$. In fact, since the $\rho_{s}\left(\theta_{1}\right)$ are analytic near $\theta_{1}=0$, and $\rho_{s}(0)=\rho_{s}^{*}$, we have, for $\theta_{1}$ in the region (2.24), $\rho_{s}=\rho_{s}^{*}+O\left(n^{-1}\right)$. Clearly $e^{ \pm i \theta_{1}}=1+O\left(n^{-1}\right)$, so

$$
\coprod_{s=2}^{b}\left(e^{ \pm i \theta_{1}}-\rho_{s}\right)^{2}=Q(1)^{2}+O\left(n^{-1}\right)
$$

and we obtain from $(2.22)$

$$
D_{1}=-2 Q(1)^{2} \sin (n+2) \theta_{1}+O\left(n^{-1}\right) .
$$

Thus if $\theta_{1}$ is a zero of $D_{1}$ we must have $\sin (n+2) \theta_{1}=O\left(n^{-1}\right)$, which implies

$$
\theta_{1}=\frac{v \pi}{n+2}+o\left(n^{-1}\right), \quad v= \pm 1, \cdots, \pm N .
$$

We have found all possible zeros, but it remains to be shown that these actually are zeros. Consider, for fixed $v$ and small $\epsilon>0$, the circle $C_{v, \epsilon}$ with center $v \pi(n+2)^{-1}$ and radius $\epsilon(n+2)^{-1}$. For a point $\theta_{1}$ on $C_{v, e}$,

$$
\theta_{1}=\frac{v \pi}{n+2}+\frac{\epsilon}{n+2} e^{i \psi}
$$

we have

$$
\left|-2 Q(1)^{2} \sin (n+2) \theta_{1}\right|=2 Q(1)^{2}\left|\sin \left(\epsilon e^{i \psi}\right)\right|>\epsilon Q(1)^{2}
$$

for sufficiently small $\epsilon$. Since the term $O\left(n^{-1}\right)$ in $(2.25)$ is $<\epsilon Q(1)^{2}$ for large enough $n$ we may apply Rouché's theorem to conclude that $D_{1}$ has inside the circle $C_{v, \epsilon}$ exactly as many zeros, counting multiplicity, as $-2 Q(1)^{2} \sin (n+2) \theta_{1}$. This function, however, has the one zero $\theta_{1}=v \pi(n+2)^{-1}$ inside $C_{v, e}$, this being a simple zero. Consequently $D_{1}$ has exactly one zero inside $C_{v, \epsilon}$, this being simple. Since $\epsilon>0$ is arbitrary we have shown that for each $v$ there is exactly one zero satisfying (2.26), this being a simple zero. (The fact that the zeros $\theta_{1}$ are simple implies that the corresponding eigenvalues $\lambda=f\left(\theta_{1}\right)$ have multiplicity one.)

To obtain a more exact expression for the zeros our evaluation of $\Pi\left(e^{ \pm i \theta_{1}}-\rho_{s}\right)^{2}$ must be more accurate. Setting $\rho_{s}=e^{i \theta_{s}}$, we have

$$
\frac{d \rho_{s}}{d \theta_{1}}=i e^{i \theta_{s}} \frac{d \theta_{s}}{d \theta}
$$

But $f\left(\theta_{s}\right)=f\left(\theta_{1}\right)(=\lambda)$ so

$$
\frac{d \theta_{s}}{d \theta_{1}} f^{\prime}\left(\theta_{s}\right)=f^{\prime}\left(\theta_{1}\right)=O\left(\left|\theta_{1}\right|\right) .
$$


Since $f^{\prime}\left(\theta_{s}\right)$ is bounded away from 0 , we conclude

$$
\frac{d \rho_{s}}{d \theta_{1}}=O\left(\left|\theta_{1}\right|\right)
$$

Thus

$$
\rho_{s}-\rho_{s}^{*}=\rho_{s}\left(\theta_{1}\right)-\rho_{s}(0)=O\left(\left|\theta_{1}\right|^{2}\right)=O\left(n^{-2}\right),
$$

from which it follows that

$$
\prod_{8=2}^{k}\left(e^{ \pm i \theta_{1}}-\rho_{s}\right)=Q\left(e^{ \pm i \theta_{1}}\right)+O\left(n^{-2}\right)
$$

Since

$$
Q\left(e^{ \pm i \theta_{1}}\right)^{2}=Q(1)^{2}+2 Q(1) Q^{\prime}(1)\left(e^{ \pm i \theta_{1}}-1\right)+O\left(n^{-2}\right),
$$

(2.22) gives

$$
\begin{aligned}
D_{1}= & -2 Q(1)^{2}\left\{\sin (n+2) \theta_{1}+2 \frac{Q^{\prime}(1)}{Q(1)}\left[\sin (n+3) \theta_{1}-\sin (n+2) \theta_{1}\right]\right\} \\
& +O\left(n^{-2}\right) \\
= & -2 Q(1)^{2}\left\{\sin (n+2) \theta_{1}+2 \frac{Q^{\prime}(1)}{Q(1)} \cos (n+2) \theta_{1} \sin \theta_{1}\right\}+O\left(n^{-2}\right) .
\end{aligned}
$$

Consider now the function

$$
D_{2}\left(\theta_{1}\right)=\sin (n+2) \theta_{1}+2 \frac{Q^{\prime}(1)}{Q(1)} \cos (n+2) \theta_{1} \sin \theta_{1}
$$

on the circle $C_{v, \mathrm{e}}^{\prime}$ with center $v \pi(n+2)^{-1}\left[1+\beta(n+2)^{-1}\right]$ and radius $\imath \pi \epsilon(n+2)^{-2}$, where $\beta$ will be determined shortly. For a point $\theta_{1}$ on $C_{v, e}^{\prime}$,

$$
\theta_{1}=\frac{v \pi}{n+2}\left[1+\frac{\beta+\epsilon e^{i \psi}}{n+2}\right],
$$

we have

$$
D_{2}\left(\theta_{1}\right)=\frac{v \pi}{n+2}(-1)^{\nu}\left[\beta+\epsilon e^{i \psi}+2 \frac{Q^{\prime}(1)}{Q(1)}\right]+O\left(n^{-2}\right) .
$$

Thus if we set

$$
\beta=-2 \frac{Q^{\prime}(1)}{Q(1)}
$$

we have 


$$
\left|-2 Q(1)^{2} D_{2}\left(\theta_{1}\right)\right|=2 Q(1)^{2} \frac{v \pi}{n+2} \epsilon+O\left(n^{-2}\right)
$$

which, for sufficiently large $n$, will be larger than the term $O\left(n^{-2}\right)$ appearing in (2.27). Again we apply Rouché's theorem to conclude that the functions $D_{1}$ and $D_{2}$ have the same number of zeros inside $C_{v, \epsilon}^{\prime}$. But $D_{2}$ is real on the real diameter of $C_{v, \varepsilon}^{\prime}$ and has opposite signs at the end-points of this diameter. Thus $D_{2}$ has a zero in $C_{v, \epsilon}^{\prime}$, and we have, for the zeros of $D_{1}$, the more exact formula

$$
\theta_{1}=\frac{v \pi}{n+2}\left(1+\frac{\beta}{n+2}\right)+o\left(n^{-2}\right), \quad v= \pm 1, \cdots, \pm N,
$$

where $\beta$ is given by $(2.28)$.

Since $\lambda=f\left(\theta_{1}\right)$ and $f(\theta)$ is even we have, for the eigenvalues,

$$
\lambda_{v, n}=M-\frac{\sigma^{2} \pi^{2} v^{2}}{2(n+1)^{2}}\left[1+\frac{\alpha}{n+1}\right]+o\left(n^{-3}\right), \quad v=1, \cdots, N,
$$

where we have set

$$
\alpha=2 \beta-2 .
$$

2.7. To evaluate $\alpha$, note that

$$
\frac{Q^{\prime}(1)}{Q(1)}=\sum \frac{1}{1-\rho_{s}^{*}}
$$

where $\rho_{s}^{*}$ runs over all the zeros of $P(z ; M)$ with absolute value greater than 1 . Since these zeros are all simple we have

$$
\frac{Q^{\prime}(1)}{Q(1)}=\frac{1}{2 \pi i} \int_{|z|=R} \frac{P^{\prime}(z ; M)}{P(z ; M)} \frac{d z}{1-z}-\frac{1}{2 \pi i} \int_{|z|=r} \frac{P^{\prime}(z ; M)}{P(z ; M)} \frac{d z}{1-z},
$$

where $r$ is sufficiently close to (but greater than) 1 and $R$ is sufficiently (and arbitrarily) large. Clearly the first integral tends to 0 as $R \rightarrow \infty$, so

$$
-\frac{Q^{\prime}(1)}{Q(1)}=\frac{1}{2 \pi i} \int_{|z|=r} \frac{P^{\prime}(z ; M)}{P(z ; M)} \frac{d z}{1-z} .
$$

We should like to transform this integral to one on the real axis. Letting $z=e^{i \theta}$ we find, from (2.12),

$$
i e^{i \theta}\left[\frac{P^{\prime}(z ; M)-k z^{-1} P(z ; M)}{P(z ; M)}\right]=\frac{f^{\prime}(\theta)}{f(\theta)-M} .
$$

It is clear, therefore, that the result of our change of variables will be simpler if we modify the integrand in (2.30). Since 


$$
\int_{|z|=r} \frac{d z}{z(1-z)}=0
$$

we may write $(2.30)$ as

$$
-\frac{Q^{\prime}(1)}{Q(1)}=\frac{1}{2 \pi i} \int_{|z|=r} \frac{P^{\prime}(z ; M)-k z^{-1} P(z ; M)}{P(z ; M)} \frac{d z}{1-z} .
$$

The integrand has a double pole at $z=1$. It follows from (2.31) that, for $z$ near 1 ,

$$
\frac{P^{\prime}(z ; M)-k z^{-1} P(z ; M)}{P(z ; M)}=\frac{4}{z^{2}-1}+O(|z-1|) .
$$

Since

$$
\int_{|z|=r} \frac{d z}{\left(z^{2}-1\right)(1-z)}=0
$$

we may write

$$
-\frac{Q^{\prime}(1)}{Q(1)}=\frac{1}{2 \pi i} \int_{|z|=r}\left[\frac{P^{\prime}(z ; M)-k z^{-1} P(z ; M)}{P(z ; M)}-\frac{4}{z^{2}-1}\right] \frac{d z}{1-z} .
$$

By (2.32) the integrand is now regular at $z=1$, but we have introduced at $z=-1$ a simple pole with residue 1 . Now deforming the contour to the unit circle we have

$$
\begin{aligned}
-\frac{Q^{\prime}(1)}{Q(1)} & =\frac{1}{2} \\
& +\frac{1}{2 \pi i} \lim _{\in \rightarrow 0} \int_{|z|=1 ;|z+1| z e}\left[\frac{P^{\prime}(z ; M)-k z^{-1} P(z ; M)}{P(z ; M)}-\frac{4}{z^{2}-1}\right] \frac{d z}{1-z} .
\end{aligned}
$$

Finally, introducing the change of variable $z=e^{i \theta}$, we have, by (2.31),

$$
\begin{aligned}
-\frac{Q^{\prime}(1)}{Q(1)} & =\frac{1}{2}+\frac{1}{2 \pi i} \lim _{\epsilon \rightarrow 0} \int_{|\theta| \leqq \pi-\epsilon}\left[\frac{f^{\prime}(\theta)}{f(\theta)-M}-2 \csc \theta\right] \frac{d \theta}{1-e^{i \theta}} \\
& =\frac{1}{2}+\frac{1}{4 \pi} \int_{-\pi}^{\pi} \cot \frac{1}{2} \theta\left[\frac{f^{\prime}(\theta)}{f(\theta)-M}-2 \csc \theta\right] d \theta .
\end{aligned}
$$

Integrating by parts and recalling (2.28) and (2.29),

$$
\alpha=\frac{1}{2 \pi} \int_{-\pi}^{\pi} \csc ^{2} \frac{1}{2} \theta \log \left[\frac{M-f(\theta)}{2 \sigma^{2}} \cot ^{2} \frac{1}{2} \theta\right] d \theta .
$$

This completes the proof of the main theorem in the special case. 
2.8. The general theorem will follow from the special case by means of approximation arguments which utilize the following

Lemma of Weyl-Courant. Denote the eigenvalues of the $n \times n$ Hermitian matrix $T$ by $\lambda_{1}, \cdots, \lambda_{n}$ (ordered in nonincreasing order). Then

$$
\lambda_{v}=\min _{Y_{1}, \ldots, Y_{v-1}} \max _{X}(T X, X)
$$

where $Y_{1}, \cdots, Y_{v-1}$ is an arbitrary set of $v-1$ vectors and $X$ runs over all vectors satisfying

$$
\|X\|=1, \quad\left(Y_{1}, X\right)=\left(Y_{2}, X\right)=\cdots=\left(Y_{v-1}, X\right)=0 .
$$

For a proof of the lemma, which is quite simple, we refer the reader to either [4, pp. 26-27] or [5, pp. 786-787].

It follows from the lemma that if we have two Hermitian matrices $T$ and $U$ such that $(T X, X) \leqq(U X, X)$ for all vectors $X$, then each eigenvalue of $T$ is less than or equal to the corresponding eigenvalue of $U$. In the case of Toeplitz matrices, we see that if we denote the eigenvalues of the $n$th Toeplitz matrix of a function $f$ by $\lambda_{v, n}(f)$ and those of the $n$th Toeplitz matrix of $g$ by $\lambda_{v, n}(g)$, then $f(\theta) \leqq g(\theta)$ for all $\theta$ implies $\lambda_{v, n}(f) \leqq \lambda_{v, n}(g)$ for all $v, n$.

2.9. Assume $f(\theta)$ is a trigonometric polynomial satisfying Condition A, but not necessarily Condition $A_{1}$. If $f(\theta)$ has degree one, it must satisfy Condition $\mathrm{A}_{1}$, so we may assume the degree of $f(\theta)$ is at least two.

For small nonzero $\epsilon$ define

$$
f_{\epsilon}(\theta)=f(\theta)+\epsilon(1-\cos \theta)^{2} .
$$

Since as $\operatorname{Im}(\theta) \rightarrow \infty,\left|f_{\epsilon}(\theta)\right| \rightarrow \infty$ uniformly for small $\epsilon$, we see that the zeros $(\bmod 2 \pi)$ of all $f_{\epsilon}(\theta)-M$ lie in a bounded set. I claim that for $\epsilon$ sufficiently small $f_{\epsilon}(\theta)-M$ will have, except for a double zero at $\theta=0$, only simple zeros. For assume we could find a sequence of $\epsilon$ 's tending to 0 , and for each $\epsilon$ a nonzero multiple zero $\theta_{\epsilon}$ of $f_{\epsilon}(\theta)-M$. Since the $\theta_{\epsilon}$ are bounded we can find, by choosing a subsequence if necessary, a $\theta_{0}$ such that $\theta_{\epsilon} \rightarrow \theta_{0}$. Then $\theta_{0}$ is a zero of $f(\theta)-M$ of multiplicity $m \geqq 2$. Note that $\theta_{0} \neq 0$, for otherwise $\theta=0$ would be a zero of $f(\theta)-M$ of multiplicity at least four.

We have

$$
f\left(\theta_{\epsilon}\right)-M=\frac{1}{m !} f^{(m)}\left(\theta_{0}\right)\left(\theta_{\epsilon}-\theta_{0}\right)^{m}+O\left(\left|\theta_{\epsilon}-\theta_{0}\right|^{m+1}\right) .
$$

Since also

$$
f_{\epsilon}\left(\theta_{\epsilon}\right)=f\left(\theta_{\epsilon}\right)+\epsilon\left(1-\cos \theta_{\epsilon}\right)^{2}=M
$$

we obtain 


$$
\left(\theta_{\epsilon}-\theta_{0}\right)^{m} \sim-\frac{m !\left(1-\cos \theta_{0}\right)^{2}}{f^{(m)}\left(\theta_{0}\right)} \epsilon \text { as } \epsilon \rightarrow 0 .
$$

But since $\theta_{\epsilon}$ is at least a double zero of $f_{\epsilon}(\theta)-M$, we have

$$
\begin{aligned}
0 & =f_{\epsilon}^{\prime}\left(\theta_{\epsilon}\right)=f^{\prime}\left(\theta_{\epsilon}\right)+2 \epsilon \sin \theta_{\epsilon}\left(1-\cos \theta_{\epsilon}\right) \\
& =\frac{1}{(m-1) !} f^{(m)}\left(\theta_{0}\right)\left(\theta_{\epsilon}-\theta_{0}\right)^{m-1}+2 \epsilon \sin \theta_{\epsilon}\left(1-\cos \theta_{\epsilon}\right)+O\left(\left|\theta_{\epsilon}-\theta_{0}\right|^{m}\right),
\end{aligned}
$$

from which we may conclude that $\left(\theta_{\epsilon}-\theta_{0}\right)^{m-1}=O(|\epsilon|)$ as $\epsilon \rightarrow 0$, which contradicts (2.33).

Thus for sufficiently small $\epsilon \neq 0, f_{\epsilon}(\theta)$ will satisfy Condition $A_{1}$. Moreover it is clear that $f_{\epsilon}(\theta)$ will satisfy Condition A with $f_{\epsilon}(0)=M$ and $-f_{\epsilon}^{\prime \prime}(0)=\sigma^{2}$. Therefore for sufficiently small $\epsilon \neq 0$ we have

$$
\lambda_{v, n}\left(f_{\epsilon}\right)=M-\frac{\sigma^{2} \pi^{2} v^{2}}{2(n+1)^{2}}\left[1+\frac{\alpha\left(f_{\epsilon}\right)}{n+1}\right]+o\left(n^{-3}\right), \quad v=1,2, \cdots .
$$

Now for $\epsilon>0, f_{-\epsilon} \leqq f \leqq f_{\epsilon}$, so by the Weyl-Courant Lemma $\lambda_{v, n}\left(f_{-\epsilon}\right)$ $\leqq \lambda_{v, n}(f) \leqq \lambda_{v, n}\left(f_{\epsilon}\right)$. Hence we shall have proved the theorem for $f(\theta)$ if we show $\alpha\left(f_{ \pm \epsilon}\right) \rightarrow \alpha(f)$ as $\epsilon \rightarrow 0$. We have

$$
\begin{aligned}
\alpha\left(f_{ \pm \epsilon}\right)-\alpha(f) & =\frac{1}{2 \pi} \int_{-\pi}^{\pi} \csc ^{2} \frac{1}{2} \theta \log \left[\frac{M-f_{ \pm \epsilon}(\theta)}{M-f(\theta)}\right] d \theta \\
& =\frac{1}{2 \pi} \int_{-\pi}^{\pi} \csc ^{2} \frac{1}{2} \theta \log \left[1 \pm \epsilon \frac{(1-\cos \theta)^{2}}{M-f(\theta)}\right] d \theta .
\end{aligned}
$$

Since $(1-\cos \theta)[M-f(\theta)]^{-1}$ is bounded, say by $c$, we have, for sufficiently small $\epsilon$,

$$
\left|\alpha\left(f_{ \pm \epsilon}\right)-\alpha(f)\right| \leqq \epsilon \frac{c}{\pi} \int_{-\pi}^{\pi} \csc ^{2} \frac{1}{2} \theta(1-\cos \theta) d \theta
$$

so $\alpha\left(f_{ \pm \epsilon}\right) \rightarrow \alpha(f)$ as $\epsilon \rightarrow 0$.

2.10. Now let $f(\theta)$ be an arbitrary function satisfying Condition A. Define

$$
g(\theta)=\frac{f(\theta)+\sigma^{2}(1-\cos \theta)-M}{(1-\cos \theta)^{2}} .
$$

Then $g(\theta)$ is even and, since $f(\theta)$ has four continuous derivatives near $\theta=0$, continuous. Therefore given $\epsilon>0$ we can find even trigonometric polynomials $g_{ \pm \epsilon}(\theta)$ satisfying

$$
g_{-\epsilon}(\theta) \leqq g(\theta) \leqq g_{\epsilon}(\theta),
$$

$$
\left|g_{ \pm \epsilon}(\theta)-g(\theta)\right|<\epsilon \quad \text { for all } \theta \text {. }
$$


Define

$$
f_{ \pm \epsilon}(\theta)=M-\sigma^{2}(1-\cos \theta)+(1-\cos \theta)^{2} g_{ \pm \epsilon}(\theta) .
$$

Since

$$
f(\theta)=M-\sigma^{2}(1-\cos \theta)+(1-\cos \theta)^{2} g(\theta)
$$

we have, by (2.34) and (2.35),

$$
\begin{aligned}
f_{-\epsilon}(\theta) & \leqq f(\theta) \leqq f_{\epsilon}(\theta), \\
\left|f_{ \pm \epsilon}(\theta)-f(\theta)\right| & \leqq \epsilon(1-\cos \theta)^{2} .
\end{aligned}
$$

We conclude readily from (2.37) that for sufficiently small $\epsilon$ the trigonometric polynomials $f_{ \pm \epsilon}(\theta)$ will satisfy Condition A with $f_{ \pm \epsilon}(0)=M$ and $-f_{ \pm \epsilon}^{\prime \prime}(0)=-\sigma^{2}$. Hence by $\S 2.9$

$$
\lambda_{v, n}\left(f_{ \pm \epsilon}\right)=M-\frac{\sigma^{2} \pi^{2} v^{2}}{2(n+1)^{2}}\left[1+\frac{\alpha\left(f_{ \pm \epsilon}\right)}{n+1}\right]+o\left(n^{-3}\right), \quad v=1,2, \cdots .
$$

Using (2.37) we may prove, as we did in $\$ 2.9$, that $\alpha\left(f_{ \pm \epsilon}\right) \rightarrow \alpha(f)$ as $\epsilon \rightarrow 0$. Thus by (2.36), the Weyl-Courant Lemma, and (2.38) we conclude the result of the theorem for $f(\theta)$.

\section{Chapter III. InTEgRal EQUations}

3.1. In this chapter we shall investigate the behavior of the extreme eigenvalues $\lambda_{v, A}$ of the integral equation (1.1), where $\rho(x) \in L_{1}$. Let $F(\xi)$ be the (automatically continuous) Fourier transform of $\rho(x)$ :

$$
F(\xi)=\int_{-\infty}^{\infty} e^{i \xi x} \rho(x) d x
$$

We shall assume either of the following conditions:

Condition B. Let $F(\xi)$ be real and even, and $O\left(|\xi|^{-2}\right)$ at infinity. Assume $\max F(\xi)=F(0)=M$ and let $\xi=0$ be the only value of $\xi$ for which this maximum is reached. Assume moreover that $F(\xi)$ has continuous derivatives up to the second order in some neighborhood of $\xi=0$, and that $\sigma^{2}=-F^{\prime \prime}(0) \neq 0$.

Condition $\mathrm{B}^{\prime}$. In addition to Condition $\mathrm{B}$, let $F(\xi)$ have continuous derivatives up to the fourth order in some neighborhood of $\xi=0$.

The main theorems of this chapter are:

Theorem 3.1. Assume $F(\xi)$ satisfies Condition B. Then as $A \rightarrow \infty$ we have, for fixed $v=1,2, \cdots$,

$$
\lambda_{v, A}=M-\frac{\sigma^{2} \pi^{2} v^{2}}{8 A^{2}}+o\left(A^{-2}\right) .
$$


Theorem 3.2. Assume $F(\xi)$ satisfies Condition $\mathrm{B}^{\prime}$. Then as $A \rightarrow \infty$ we have, for fixed $v=1,2, \cdots$,

$$
\lambda_{v, A}=M-\frac{\sigma^{2} \pi^{2} v^{2}}{8 A^{2}}\left[1+\frac{\alpha}{A}\right]+o\left(A^{-3}\right),
$$

where

$$
\alpha=\alpha(F)=\frac{1}{\pi} \int_{-\infty}^{\infty} \frac{1}{\xi^{2}} \log \left[\frac{2}{\sigma^{2}} \frac{M-F(\xi)}{\xi^{2}}\right] d \xi .
$$

3.2. We shall prove the theorems first under the assumption that $F(\xi)$ is a rational function (in which case Conditions $\mathrm{B}$ and $\mathrm{B}^{\prime}$ coincide) and satisfies the additional conditions:

Condition $\mathrm{B}_{1}$. All poles of $F(\xi)$ are simple.

Condition $\mathrm{B}_{2}$. All zeros of $F(\xi)-M$, with the exception of the double zero at $\xi=0$, are simple.

By the Fourier inversion formula

$$
\rho(x)=\frac{1}{2 \pi} \int_{-\infty}^{\infty} e^{-i \xi x} F(\xi) d \xi,
$$

the integral equation (1.1) may be written

$$
\frac{1}{2 \pi} \int_{-\infty}^{\infty} e^{-i \xi x} F(\xi) d \xi \int_{-A}^{A} e^{i \xi y} \phi(y) d y=\lambda \phi(x) .
$$

If we write

$$
F(\xi)=P(\xi) / Q(\xi),
$$

where $P(\xi)$ and $Q(\xi)$ are polynomials with no common factor, it is well known that (3.1) can be reduced to the differential equation written symbolically as

$$
P\left(i \frac{d}{d x}\right) \phi(x)=\lambda Q\left(i \frac{d}{d x}\right) \phi(x) .
$$

The solution of (3.2) depends on the zeros of $F(\xi)-\lambda$. Now we may restrict our attention to those $\lambda$ which lie in an arbitrarily small open interval $(M, M-\delta)$. This is seen as follows. Let $\phi(x)$ be a solution of (3.1) normalized so that

$$
2 \pi \int_{-A}^{A}|\phi(x)|^{2} d x=1
$$

Then multiplying (3.1) by $\phi(x)$ and integrating we obtain 


$$
\lambda=\int_{-\infty}^{\infty} F(\xi)|\Phi(\xi)|^{2} d \xi
$$

where we have set

$$
\Phi(\xi)=\int_{-A}^{A} e^{i \xi x} \phi(x) d x
$$

and used Parseval's theorem. Since $\int_{-\infty}^{\infty}|\Phi(\xi)|^{2} d \xi=1$, it is clear by Condition $\mathrm{B}$ that $\lambda<M$. Also, since each $\lambda_{v, A} \rightarrow M$ as $A \rightarrow \infty$, it is clear that nothing will be lost if we assume $\lambda \in(M, M-\delta)$, where $\delta$ is as small as we please.

Now for sufficiently small $\delta$ and $\lambda \in(M, M-\delta), F(\xi)-\lambda$ will have only simple zeros; for if we denote by $\left\{\xi^{\prime}\right\}$ the (finite) set of zeros of $F^{\prime}(\xi)$ we need only choose $\delta$ so that none of the quantities $F\left(\xi^{\prime}\right)$ lie in $(M, M-\delta)$. Denoting the zeros of $F(\xi)-\lambda$ by $\xi_{1}, \cdots, \xi_{2 q}$ (where $2 q$ is the degree of $Q$ ) the general solution of (3.2) may be written

$$
\phi(x)=\sum_{j=1}^{2 q} c_{j} e^{i \xi_{j} x}
$$

If we substitute this expression into (3.1), the integral can be calculated explicitly. Let the poles of $F(\xi)$ in the upper half-plane be $\eta_{1}, \cdots, \eta_{q}$; then the poles in the lower half-plane will be $-\eta_{1}, \cdots,-\eta_{q}$. Remembering that $|x| \leqq A$, (3.1) becomes

$$
0=\sum_{k=1}^{q} r_{k} e^{i A \eta_{k}}\left(\sum_{j=1}^{2 q} \frac{c_{j} e^{i A \xi_{j}}}{\xi_{j}+\eta_{k}}\right) e^{-i \eta_{k} x}+\sum_{k=1}^{q} r_{k} e^{i A \eta_{k}}\left(\sum_{j=1}^{2 q} \frac{c_{j} e^{-i A \xi_{j}}}{\xi_{j}-\eta_{k}}\right) e^{i \eta_{k} x},
$$

where $r_{k}$ is the residue of $F(\xi)$ at $\xi=\eta_{k}$. Since the functions $e^{i \eta_{1} x}, \cdots, e^{i \eta x}$, $e^{-i \eta_{1} x}, \cdots, e^{-i \eta_{q} x}$ are linearly independent, (3.5) is equivalent to the system of equations

$$
\begin{array}{ll}
\sum_{j=1}^{2 q} c_{j} \frac{e^{i A \xi_{j}}}{\xi_{j}+\eta_{k}}=0, & k=1, \cdots, q, \\
\sum_{j=1}^{2 q} c_{j} \frac{e^{-i A \xi_{i}}}{\xi_{j}-\eta_{k}}=0, & k=1, \cdots, q .
\end{array}
$$

We shall change our notation slightly. The function $F(\xi)-M$ has $2 q-2$ complex zeros, $q-1$ in the upper half-plane and $q-1$ in the lower. Denote the zeros in the upper half-plane by $\xi_{2}^{*}, \cdots, \xi_{q}^{*}$; then the remaining complex zeros are $-\xi_{2}^{*}, \cdots,-\xi_{q}^{*}$. By Condition $\mathrm{B}, F(\xi)-\lambda$ will have, for $\lambda \in(M, M-\delta)$, two real zeros which we denote by $\xi_{1}$ and $-\xi_{1}$, and $2 q-2$ complex zeros $\pm \xi_{2}, \cdots, \pm \xi_{q}$, with $\xi_{j} \rightarrow \xi_{j}^{*}$ as $\lambda \rightarrow M$. Using this new notation for the zeros of $F(\xi)-\lambda$ we see that the multiplicity of $\lambda$ is the number of linearly independent solutions of (3.6) for $c_{1}, \cdots, c_{2 q}$, or equivalently $2 q$ minus the rank of the matrix 


$$
\left(\begin{array}{cccccc}
\frac{e^{i A \xi_{1}}}{\xi_{1}+\eta_{1}} & \frac{e^{-i A \xi_{1}}}{-\xi_{1}+\eta_{1}} & \frac{e^{i A \xi_{2}}}{\xi_{2}+\eta_{1}} & \frac{e^{-i A \xi_{2}}}{-\xi_{2}+\eta_{1}} \cdots \frac{e^{i A \xi_{q}}}{\xi_{q}+\eta_{1}} & \frac{e^{-i A \xi_{q}}}{-\xi_{q}+\eta_{1}} \\
\vdots & \vdots & \vdots & \vdots & \vdots & \vdots \\
\frac{e^{i \dot{A} \xi_{1}}}{\xi_{1}+\eta_{q}} & \frac{e^{-i A \xi_{1}}}{-\xi_{1}+\eta_{q}} & \frac{e^{i \dot{A} \xi_{2}}}{\xi_{2}+\eta_{q}} & \frac{e^{-i A \xi_{2}}}{-\xi_{2}+\eta_{q}} \cdots \frac{e^{i A \xi_{q}}}{\xi_{q}+\eta_{q}} & \frac{e^{-i \Delta \xi_{q}}}{-\xi_{q}+\eta_{q}} \\
\frac{e^{-i A \xi_{1}}}{\xi_{1}-\eta_{1}} & \frac{e^{i A \xi_{1}}}{-\xi_{1}-\eta_{1}} & \frac{e^{-i A \xi_{2}}}{\xi_{2}-\eta_{1}} & \frac{e^{i A \xi_{2}}}{-\xi_{2}-\eta_{1}} \cdots \frac{e^{-i A \xi_{q}}}{\xi_{q}-\eta_{1}} & \frac{e^{i A \xi_{q}}}{-\xi_{q}-\eta_{1}} \\
\vdots & \vdots & \vdots & \vdots & \vdots & \vdots \\
\frac{e^{-i A \xi_{1}}}{\xi_{1}-\eta_{q}} & \frac{e^{i A \xi_{1}}}{-\xi_{1}-\eta_{q}} & \frac{e^{-i A \xi_{2}}}{\xi_{2}-\eta_{q}} & \frac{e^{i A \xi_{2}}}{-\xi_{2}-\eta_{q}} \cdots \frac{e^{-i A \xi_{q}}}{\xi_{q}-\eta_{q}} & \frac{e^{i \dot{A} \xi_{q}}}{-\xi_{q}-\eta_{q}}
\end{array}\right)
$$

3.3. Denote by $D$ the determinant of the matrix (3.7), and write

$$
D_{1}=D \exp \left(2 i A \sum_{j=2}^{q} \xi_{j}\right)
$$

Then we have

$$
D_{1}=\left|\begin{array}{cccccc}
\frac{e^{i A \xi_{1}}}{\xi_{1}+\eta_{1}} & \frac{e^{-i A \xi_{1}}}{-\xi_{1}+\eta_{1}} & \frac{e^{2 i A \xi_{2}}}{\xi_{2}+\eta_{1}} & \frac{1}{-\xi_{2}+\eta_{1}} \cdots & \cdots \frac{e^{2 i A \xi_{q}}}{\xi_{q}+\eta_{1}} & \frac{1}{-\xi_{q}+\eta_{1}} \\
\vdots & \vdots & \vdots & \vdots & \vdots & \vdots \\
\frac{e^{i A \xi_{1}}}{\xi_{1}+\eta_{q}} & \frac{e^{-i A \xi_{1}}}{-\xi_{1}+\eta_{q}} & \frac{e^{2 i A \xi_{2}}}{\xi_{2}+\eta_{q}} & \frac{1}{\xi_{2}+\eta_{q}} \cdots & \cdots \frac{e^{2 i A \xi_{q}}}{\xi_{q}+\eta_{q}} & \frac{1}{-\xi_{q}+\eta_{q}} \\
\frac{e^{-i A \xi_{1}}}{\xi_{1}-\eta_{1}} & \frac{e^{i A \xi_{1}}}{-\xi_{1}-\eta_{1}} & \frac{1}{\xi_{2}-\eta_{1}} & \frac{e^{2 i A \xi_{2}}}{-\xi_{2}-\eta_{1}} \cdots \frac{1}{\xi_{q}-\eta_{1}} & \frac{e^{2 i A \xi_{q}}}{-\xi_{q}-\eta_{1}} \\
\vdots & \vdots & \vdots & \vdots & \vdots & \vdots \\
\frac{e^{-i A \xi_{1}}}{\xi_{1}-\eta_{q}} & \frac{e^{i A \xi_{1}}}{-\xi_{1}-\eta_{q}} & \frac{\dot{1}}{\xi_{2}-\eta_{q}} & \frac{e^{2 i A \xi_{2}}}{-\xi_{2}-\eta_{q}} \cdots \frac{1}{\xi_{q}-\eta_{q}} & \frac{e^{2 i A \xi_{q}}}{-\xi_{q}-\eta_{q}}
\end{array}\right|
$$

Now for $\lambda$ close to $M$, the various $\xi_{j}(j=2, \cdots, q)$ will be close to the corresponding $\xi_{j}^{*}$. If we set

$$
2 \mu=\min _{j=2, \cdots, q} \operatorname{Im}\left(\xi_{j}^{*}\right)>0,
$$

then for $\lambda$ sufficiently close to $M$ we shall have

$$
\operatorname{Im}\left(\xi_{j}\right) \geqq \mu, \quad j=2, \cdots, q .
$$

Thus, in the expansion of $D_{1}$ any term involving $e^{2 i A \xi_{i}}$ is $O\left(e^{-2 A \mu}\right)$, so we may write 


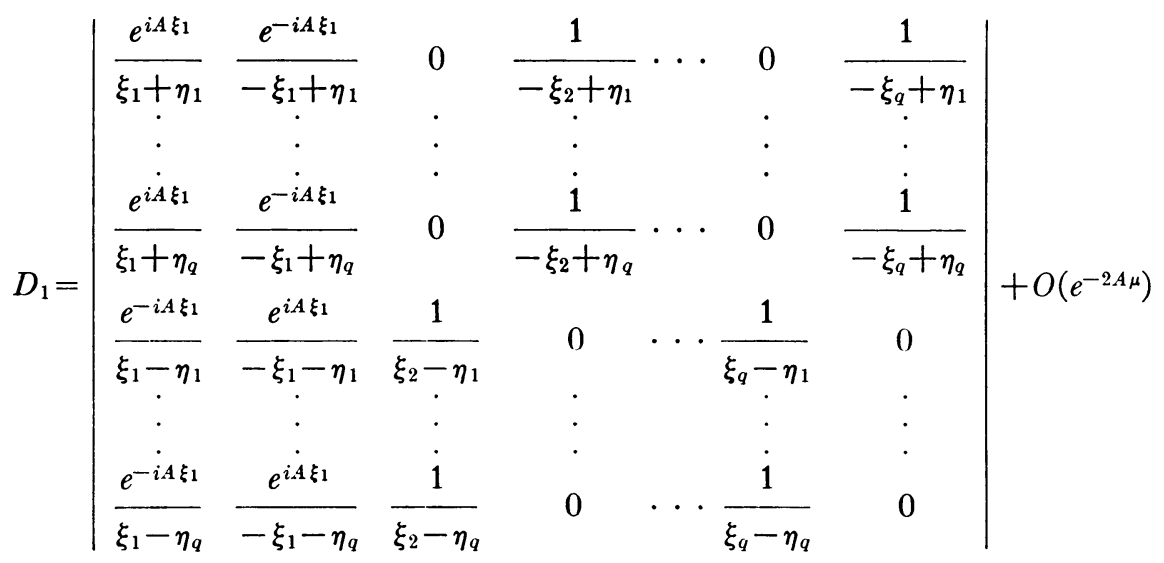

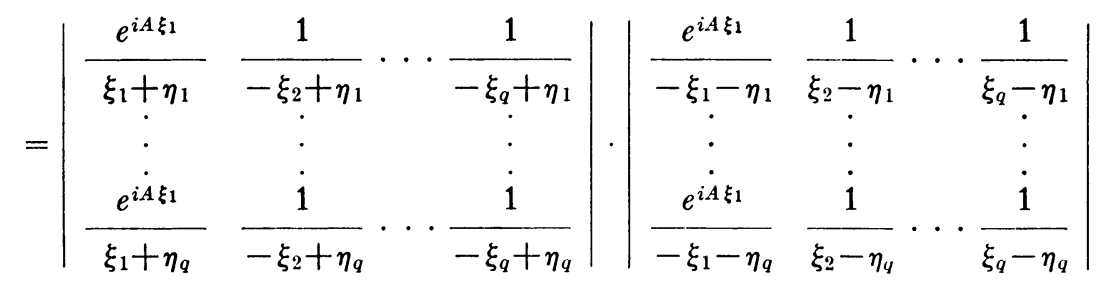

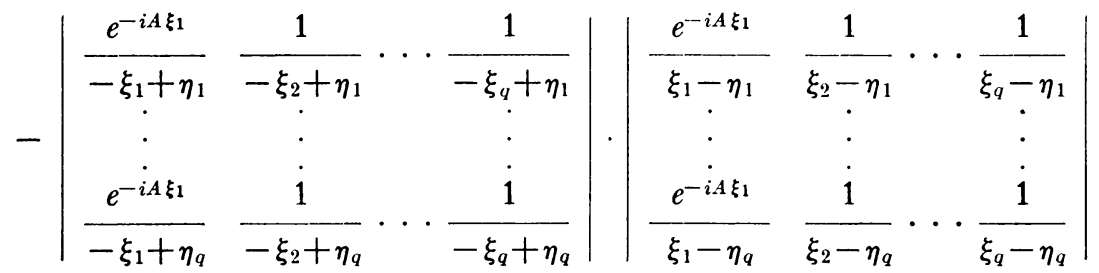

$$
+O\left(e^{-2 A \mu}\right)
$$

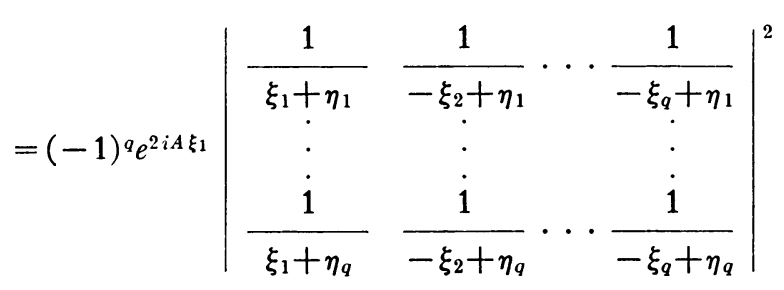$$
-(-1)^{q} e^{-2 i A \xi_{1}}\left|\begin{array}{ccc}
\frac{1}{\xi_{1}-\eta_{1}} & \frac{1}{\xi_{2}-\eta_{1}} \cdots \frac{1}{\xi_{q}-\eta_{1}} \\
\vdots & \vdots & \vdots \\
\frac{1}{\xi_{1}-\eta_{q}} & \frac{i}{\xi_{2}-\eta_{q}} \cdots \frac{1}{\xi_{q}-\eta_{q}}
\end{array}\right|^{2}+O\left(e^{-2 A \mu}\right)
$$ 
The two determinants obtained are both cases of the Cauchy determinant

$$
\left|\frac{1}{a_{j}+b_{k}}\right|
$$$$
j, k=1, \cdots, q \text {, }
$$

whose value is

$$
\frac{\prod_{j<k}\left(a_{j}-a_{k}\right)\left(b_{j}-b_{k}\right)}{\prod_{j, k}\left(a_{j}+b_{k}\right)} .
$$

(See, for example, [6, pp. 87-88].) The calculation is therefore finished. If we set

$$
D_{2}=(-1)^{q} \frac{\prod_{j \geqq 2 ; k \geqq 1}\left(\xi_{j}-\eta_{k}\right)^{2}}{\prod_{2 \leq j<k}\left(\xi_{j}-\xi_{k}\right)^{2} \prod_{1 \leq j<k}\left(\eta_{j}-\eta_{k}\right)^{2}} D_{1},
$$

then

$$
D_{2}=e^{2 i A \xi_{1}} \frac{\prod_{k \geq 2}\left(\xi_{1}+\xi_{k}\right)^{2}}{\prod_{k \geq 1}\left(\xi_{1}+\eta_{k}\right)^{2}}-e^{-2 i A \xi_{1}} \frac{\prod_{k \geq 2}\left(\xi_{1}-\xi_{k}\right)^{2}}{\prod_{k \geq 1}\left(\xi_{1}-\eta_{k}\right)^{2}}+O\left(e^{-2 A \mu}\right) .
$$

3.4. In calculating the zeros of $D_{2}$ we shall have to know that the "error" term $O\left(e^{-2 \Lambda \mu}\right)$ appearing in (3.8) is much smaller than the remaining "dominant" term. It is clear, however, that for $M-\lambda$ of the order $e^{-4 A \mu}$, or equivalently $\xi_{1}$ of the order $e^{-2 A \mu}$, the dominant term is roughly the same size as the error term. We eliminate this difficulty by showing that no eigenvalue can get nearly this close to $M$.

Lemma. There is a constant $a>0$ such that if $\lambda$ is an eigenvalue of (1.1) with $A$ sufficiently large, then $\lambda \leqq M-a A^{-2}$.

To prove the lemma, we shall use (3.3) more effectively than was done to show merely that $\lambda<M$. For sufficiently small $\xi$, we have

$$
F(\xi) \leqq M-\frac{1}{4} \sigma^{2} \xi^{2} \quad\left[\sigma^{2}=-F^{\prime \prime}(0)\right],
$$

and, by Condition B,

$$
\begin{aligned}
\max _{|\xi| \geqq A^{-1}} F(\xi)=F\left(A^{-1}\right) \\
\leqq M-\frac{1}{4} \sigma^{2} A^{-2}
\end{aligned}
$$

for sufficiently large $A$. Recalling that $\int_{-\infty}^{\infty}|\Phi(\xi)|^{2} d \xi=1$ we obtain from (3.3), 


$$
\lambda \leqq \int_{-A^{-1}}^{A^{-1}}\left(M-\frac{1}{4} \sigma^{2} \xi^{2}\right)|\Phi(\xi)|^{2} d \xi+\left(M-\frac{1}{4} \sigma^{2} A^{-2}\right) \int_{|\xi| \geqq A^{-1}}|\Phi(\xi)|^{2} d \xi
$$

$$
\begin{aligned}
& =M-\frac{1}{4} \sigma^{2}\left(\int_{-A^{-1}}^{A^{-1}} \xi^{2}|\Phi(\xi)|^{2} d \xi+A^{-2} \int_{|\xi| \geq A^{-1}}|\Phi(\xi)|^{2} d \xi\right) \\
& =M-\frac{1}{4} \sigma^{2}\left(A^{-2}-\int_{-A^{-1}}^{A^{-1}}\left(A^{-2}-\xi^{2}\right)|\Phi(\xi)|^{2} d \xi\right) .
\end{aligned}
$$

Recalling that $2 \pi \int_{-A}^{A}|\phi(x)|^{2} d x=1$ and applying Schwarz's inequality to (3.4) we obtain $|\Phi(\xi)|^{2} \leqq A / \pi$ for all $\xi$, so

$$
\int_{-A^{-1}}^{A^{-1}}\left(A^{-2}-\xi^{2}\right)|\Phi(\xi)|^{2} d \xi \leqq \frac{4}{3 \pi} A^{-2}
$$

and (3.9) gives

$$
\lambda \leqq M-\frac{1}{4} \sigma^{2}\left(1-\frac{4}{3 \pi}\right) A^{-2} .
$$

3.5. It is convenient now to consider $D, D_{1}$ and $D_{2}$ as functions of the complex variable $\xi_{1}$. For $\xi_{1}$ sufficiently close to $0, \lambda=F\left(\xi_{1}\right)$ is close to $M$, and the zeros $\pm \xi_{2}, \cdots, \pm \xi_{q}$, being analytic functions of $\lambda\left( \pm \xi_{2}^{*}, \cdots, \pm \xi_{q}^{*}\right.$ are simple zeros of $F(\xi)-M$ ), are also analytic functions of $\xi_{1}$. Thus $D$, the determinant of the matrix (3.7) is analytic in some region $\left|\xi_{1}\right|<\delta$, and $D_{1}$ and $D_{2}$ are analytic in the same region.

We know from the lemma of $\$ 3.4$ that $D_{2}$ is free of zeros in some region $\left|\xi_{1}\right| \leqq b A^{-1}$. We shall confine our attention to the ring

$$
\frac{b}{A}<\left|\xi_{1}\right|<\frac{(N+1 / 2) \pi}{2 A}
$$

where $N$ is arbitrarily large but fixed, and we make certain that $b<\pi / 2$. We shall show that $D_{2}\left(\xi_{1}\right)$ has exactly $2 N$ zeros in the region (3.10), these being simple.

We note first that the quantities $\xi_{k}$ appearing in (3.8) may be replaced by $\xi_{\boldsymbol{k}}^{*}$ without too much of an error. Since $F\left(\xi_{k}\right)=F\left(\xi_{1}\right)(=\lambda)$,

$$
F^{\prime}\left(\xi_{k}\right) \frac{d \xi_{k}}{d \xi_{1}}=F^{\prime}\left(\xi_{1}\right)=O\left(A^{-1}\right)
$$

for $\xi_{1}$ in the region $(3.10)$, so $d \xi_{k} / d \xi_{1}=O\left(A^{-1}\right)$. Therefore, since $\xi_{\mathfrak{k}}^{*}=\xi_{k}(0)$, $\xi_{k}=\xi_{\mathbf{k}}^{*}+O\left(A^{-2}\right)$. Thus if we introduce the rational function

$$
G(\xi)=\frac{\prod_{k \geq 2}\left(\xi-\xi_{k}^{*}\right)}{\prod_{k \geq 1}\left(\xi-\eta_{k}\right)}
$$


we obtain from (3.8),

$$
D_{2}=G\left(-\xi_{1}\right)^{2} e^{2 i A \xi_{1}}-G\left(\xi_{1}\right)^{2} e^{-2 i A \xi_{1}}+O\left(A^{-2}\right) .
$$

Since $G\left( \pm \xi_{1}\right)=G(0)+O\left(A^{-1}\right)$ we obtain for a first approximation

$$
D_{2}=2 i G(0)^{2} \sin 2 A \xi_{1}+O\left(A^{-1}\right) .
$$

Thus if $\xi_{1}$ is a zero of $D_{2}$ we must have $\sin 2 A \xi_{1}=O\left(A^{-1}\right)$, which implies

$$
\xi_{1}=\frac{v \pi}{2 A}+o\left(A^{-1}\right), \quad v= \pm 1, \cdots, \pm N .
$$

We have located all possible zeros of $D_{2}$ in the region (3.10), but it remains to be shown that these actually are zeros. Consider, for fixed $v$ and small $\epsilon>0$, the circle $C_{v, \epsilon}$ with center $v \pi / 2 A$ and radius $\epsilon / 2 A$. For a point $\xi_{1}$ on $C_{v, \epsilon}$,

$$
\xi_{1}=\frac{v \pi}{2 A}+\frac{\epsilon}{2 A} e^{i \psi}
$$

we have

$$
\text { | } 2 i G(0)^{2} \sin 2 A \xi_{1}\left|=2 G(0)^{2}\right| \sin \left(\epsilon e^{i \psi}\right) \mid>\epsilon G(0)^{2}
$$

for sufficiently small $\epsilon$. Since the term $O\left(A^{-1}\right)$ appearing in (3.12) is $<\epsilon G(0)^{2}$ for large enough $A$ we may apply Rouché's theorem to conclude that inside $C_{v, e}$ the functions $D_{2}\left(\xi_{1}\right)$ and $2 i G(0)^{2} \sin 2 A \xi_{1}$ have the same number of zeros, counting multiplicity. The latter function, however, has the one zero $\xi_{1}$ $=v \pi / 2 A$ inside $C_{v, \epsilon}$, this being a simple zero. Consequently $D_{2}$ has exactly one zero, this being a simple one, inside $C_{v, \epsilon}$. Since $\epsilon>0$ was arbitrary, we have shown that for each $v$ there is exactly one zero of $D_{2}$ satisfying (3.13), this being a simple zero. (The fact that the zeros $\xi_{1}$ are simple implies that the corresponding eigenvalues $\lambda=F\left(\xi_{1}\right)$ have multiplicity one.)

To obtain the next order of approximation, we note that

$$
G\left( \pm \xi_{1}\right)^{2}=G(0)^{2} \pm 2 G^{\prime}(0) G(0) \xi_{1}+O\left(\left|\xi_{1}\right|^{2}\right),
$$

so (3.11) becomes

$$
D_{2}=2 i G(0)^{2}\left\{\sin 2 A \xi_{1}+2 i \frac{G^{\prime}(0)}{G(0)} \cos 2 A \xi_{1} \sin \xi_{1}\right\}+O\left(A^{-2}\right)
$$

Consider now the function

$$
D_{3}\left(\xi_{1}\right)=\sin 2 A \xi_{1}+2 i \frac{G^{\prime}(0)}{G(0)} \cos 2 A \xi_{1} \sin \xi_{1}
$$

on the circle $C_{v, \epsilon}^{\prime}$ with center $v \pi(2 A)^{-1}\left[1+\alpha(2 A)^{-1}\right]$ and radius $v \pi \epsilon(2 A)^{-2}$, where $\alpha$ will be determined shortly. For a point $\xi_{1}$ on $C_{v, \epsilon}^{\prime}$, 


$$
\xi_{1}=\frac{v \pi}{2 A}\left[1+\frac{\alpha+\epsilon e^{i \psi}}{2 A}\right]
$$

we have

$$
D_{3}\left(\xi_{1}\right)=\frac{v \pi}{2 A}(-1)^{v}\left\{\alpha+\epsilon e^{i \psi}+2 i \frac{G^{\prime}(0)}{G(0)}\right\}+O\left(A^{-2}\right) .
$$

Thus if we set

$$
\alpha=-2 i \frac{G^{\prime}(0)}{G(0)}
$$

we have

$$
D_{3}\left(\xi_{1}\right)=(-1)^{v} v \pi i \frac{G^{\prime}(0)}{G(0)} \frac{\epsilon e^{i \psi}}{A}+O\left(A^{-2}\right) .
$$

Again we may apply Rouché's theorem to conclude from (3.14) that the functions $D_{2}$ and $D_{3}$ have the same number of zeros inside $C_{v, \epsilon}^{\prime}$. Now since

$$
\frac{G^{\prime}(0)}{G(0)}=-\sum_{k=2}^{q} \frac{1}{\xi_{k}^{*}}+\sum_{k=1}^{q} \frac{1}{\eta_{k}}
$$

is purely imaginary, it is clear from (3.15) that $D_{3}$ is real for real $\xi_{1}$. Since, by (3.17), $D_{3}$ has opposite signs at the ends of the real diameter of $C_{v, e}^{\prime}$, we see that $D_{3}$, and so also $D_{2}$, has a zero inside $C_{v, \epsilon}^{\prime}$. Therefore we have the following more exact expression for the zeros:

$$
\xi_{1}=\frac{v \pi}{2 A}\left[1+\frac{\alpha}{2 A}\right]+o\left(A^{-2}\right), \quad v= \pm 1, \pm 2, \cdots,
$$

where $\alpha$ is given by (3.16).

Since $\lambda=F\left(\xi_{1}\right)$ and $F(\xi)$ is even we have, for the eigenvalues,

$$
\lambda_{v, A}=M-\frac{\sigma^{2} \pi^{2} v^{2}}{8 A^{2}}\left[1+\frac{\alpha}{A}\right]+o\left(A^{-3}\right), \quad v=1,2, \cdots .
$$

3.6. To complete the proof of Theorem 3.2 in our special case, we have to evaluate $\alpha$. Noting that the $\xi_{k}^{*}$ and $\eta_{k}$ in (3.18) are, respectively, the zeros and poles (all simple) of $F(\xi)-M$ in the upper half-plane, (3.16) and (3.18) give

$$
\alpha=\frac{1}{\pi} \int_{C} \frac{F^{\prime}(\xi)}{F(\xi)-M} \frac{d \xi}{\xi},
$$

where $C$ is any contour, described in the positive direction, enclosing all the $\xi_{\boldsymbol{k}}^{*}$ and $\eta_{k}$ but not touching the real axis. Now the integrand has a pole at 
$\xi=0$ with principal part $2 \xi^{-2}$. Hence

$$
\left[\frac{F^{\prime}(\xi)}{F(\xi)-M}-\frac{2}{\xi}\right] \frac{1}{\xi}
$$

is regular on the real axis. Moreover $\int_{C} \xi^{-2} d \xi=0$. Therefore we can write

$$
\alpha=\frac{1}{\pi} \int_{C^{\prime}}\left[\frac{F^{\prime}(\xi)}{F(\xi)-M}-\frac{2}{\xi}\right] \frac{d \xi}{\xi},
$$

where $C^{\prime}$ consists of the real axis from $-R$ to $R$ and the upper half of the circle with center $\xi=0$ and radius $R$. As $R \rightarrow \infty$ the integral over the second portion of $C^{\prime}$ approaches zero, so

$$
\alpha=\frac{1}{\pi} \int_{-\infty}^{\infty}\left[\frac{F^{\prime}(\xi)}{F(\xi)-M}-\frac{2}{\xi}\right] \frac{d \xi}{\xi} .
$$

Finally, integration by parts yields

$$
\alpha=\frac{1}{\pi} \int_{-\infty}^{\infty} \frac{1}{\xi^{2}} \log \left[\frac{2}{\sigma^{2}} \frac{M-F(\xi)}{\xi^{2}}\right] d \xi .
$$

3.7. The general Theorems 3.1 and 3.2 will follow from the special case by use of approximations which utilize the following

Lemma of Weyl-Courant. Denote the positive eigenvalues of the integral equation

$$
\int \rho(x, y) \phi(y)=\lambda \phi(x), \quad \rho(x, y)=\rho(y, x)
$$

by $\lambda_{1}, \lambda_{2}, \cdots$ (ordered in nonincreasing order). Then

$$
\lambda_{\nu}=\min _{\psi_{1}, \ldots, \psi_{v-1}} \max _{\phi} \iint \rho(x, y) \phi(x) \phi(y) d x d y
$$

where $\psi_{1}(x), \cdots, \psi_{v-1}(x)$ are arbitrary and $\phi(x)$ runs over all functions satisfying

$$
\int|\phi(x)|^{2} d x=1, \quad \int \psi_{i}(x) \phi(x) d x=0, \quad(i=1, \cdots, v-1) .
$$

For a proof of this result, see [4, pp. 112-113].

It follows immediately from the Weyl-Courant Lemma that if we have a second symmetric kernel $\rho_{1}(x, y)$ such that $\iint \rho(x, y) \phi(x) \phi(y) d x d y$ $\leqq \iint \rho_{1}(x, y) \phi(x) \phi(y) d x d y$ for all $\phi$, then each positive eigenvalue of the integral equation with kernel $\rho$ is less than or equal to the corresponding eigenvalue of the integral equation with kernel $\rho_{1}$. For the equations we are con- 
cerned with, this may be interpreted as follows: Let $\rho(x)$ have the Fourier transform $F(\xi)$ and $\rho_{1}(x)$ the Fourier transform $F_{1}(\xi)$. Denote the positive eigenvalues of

$$
\int_{-A}^{A} \rho(x-y) \phi(y) d y=\lambda \phi(x)
$$

by $\lambda_{1, A}(F) \geqq \lambda_{2, A}(F) \geqq \cdots$, , and those of

$$
\int_{-A}^{A} \rho_{1}(x-y) \phi(y) d y=\lambda \phi(x)
$$

by $\lambda_{1, A}\left(F_{1}\right) \geqq \lambda_{2, A}\left(F_{1}\right) \geqq \cdots$. Then if $F(\xi) \leqq F_{1}(\xi)$ for all $\xi$ we have $\lambda_{v, A}(F)$ $\leqq \lambda_{v, A}\left(F_{1}\right)$ for all $v, A$.

3.8. Assume $F(\xi)$ is rational and satisfies Conditions $B$ and $B_{1}$, but not necessarily $B_{2}$. For small nonzero $\epsilon$ define

$$
F_{\epsilon}(\xi)=F(\xi)+\epsilon \frac{\xi^{4}}{1+\xi^{6}} .
$$

It is clear that for sufficiently small $\epsilon, F_{\epsilon}(\xi)$ will satisfy Condition B with $F_{\epsilon}(0)=M$ and $-F_{\epsilon}^{\prime \prime}(0)=\sigma^{2}$ and also Condition $\mathrm{B}_{1}$. I claim that $F_{\epsilon}(\xi)$ will also satisfy Condition $B_{2}$. Assuming the contrary we can find a sequence of $\epsilon$ 's tending to zero and for each $\epsilon$ a nonzero multiple zero $\xi_{\epsilon}$ of $F_{\epsilon}(\xi)-M$. Since the $\xi_{\epsilon}$ are bounded (note that as $\xi \rightarrow \infty, F_{\epsilon}(\xi)-M \rightarrow-M$ uniformly in $\epsilon$ ) we can find, by choosing a subsequence if necessary, a point $\xi_{0}$ such that $\xi_{\epsilon} \rightarrow \xi_{0}$. Then $\xi_{0}$ is a zero of $F(\xi)-M$ of multiplicity $m \geqq 2$. Moreover $\xi_{0} \neq 0$, for otherwise $\xi=0$ would be a zero of $F(\xi)-M$ of multiplicity at least four. From

$$
F_{\epsilon}\left(\xi_{\epsilon}\right)=F\left(\xi^{1}\right)+\epsilon \frac{\xi_{\epsilon}^{4}}{1+\xi_{\epsilon}^{6}}=M
$$

and

$$
F\left(\xi_{\epsilon}\right)=M+\frac{1}{m !} F^{(m)}\left(\xi_{0}\right)\left(\xi_{\epsilon}-\xi_{0}\right)^{m}+O\left(\left|\xi_{\epsilon}-\xi_{0}\right|^{m+1}\right)
$$

we conclude

$$
\left(\xi_{\epsilon}-\xi_{0}\right)^{m} \sim-\frac{m ! \xi_{0}^{4}}{F^{(m)}\left(\xi_{0}\right)\left(1+\xi_{0}^{6}\right)} \epsilon \text { as } \epsilon \rightarrow 0 .
$$

Similarly from $F_{\epsilon}^{\prime}\left(\xi_{\epsilon}\right)=0$ we conclude $\left(\xi_{\epsilon}-\xi_{0}\right)^{m-1}=O(|\epsilon|)$, which contradicts (3.19).

Thus for sufficiently small $\epsilon, F_{\epsilon}(\xi)$ will satisfy all the conditions under 
which we have proved Theorem 3.2. If, for sufficiently small positive $\epsilon$, we define

$$
\rho_{ \pm \epsilon}(x)=\frac{1}{2 \pi} \int_{-\infty}^{\infty} e^{-i \xi x} F_{ \pm \epsilon}(\xi) d \xi
$$

we have the Fourier inversion formula

$$
F_{ \pm \epsilon}(\xi)=\int_{-\infty}^{\infty} e^{i \xi x} \rho_{ \pm \epsilon}(x) d x
$$

and

$$
\lambda_{v, A}\left(F_{ \pm \epsilon}\right)=M-\frac{\sigma^{2} \pi^{2} v^{2}}{8 A^{2}}\left[1+\frac{\alpha\left(F_{ \pm \epsilon}\right)}{A}\right]+o\left(A^{-3}\right), \quad v=1,2, \cdots .
$$

Now since $F_{-\epsilon}(\xi) \leqq F(\xi) \leqq F_{\epsilon}(\xi)$ we have, by the Lemma of Weyl-Courant, $\lambda_{v, A}\left(F_{-\epsilon}\right) \leqq \lambda_{v, A}(F) \leqq \lambda_{v, A}\left(F_{\epsilon}\right)$. Therefore we shall have proved Theorem 3.2 for $F(\xi)$ if we show $\alpha\left(F_{ \pm \epsilon}\right) \rightarrow \alpha(F)$ as $\epsilon \rightarrow 0$. We have

$$
\alpha\left(F_{ \pm \epsilon}\right)-\alpha(F)=\frac{1}{\pi} \int_{-\infty}^{\infty} \frac{1}{\xi^{2}} \log \left[1+\frac{F(\xi)-F_{ \pm \epsilon}(\xi)}{M-F(\xi)}\right] d \xi .
$$

Now $[M-F(\xi)]^{-1}$ is $O\left(|\xi|^{-2}\right)$ near $\xi=0$ and bounded at infinity, so for some constant $c>0$ we have

$$
\frac{F(\xi)-F_{ \pm \epsilon}(\xi)}{M-F(\xi)} \leqq \epsilon \frac{c^{2}}{1+\xi^{4}} .
$$

Therefore for sufficiently small $\epsilon$

$$
\left|\alpha\left(F_{ \pm \epsilon}\right)-\alpha(F)\right| \leqq \epsilon \frac{2 c}{\pi} \int_{-\infty}^{\infty} \frac{d \xi}{1+\xi^{4}}
$$

which shows $\alpha\left(F_{ \pm \epsilon}\right) \rightarrow \alpha(F)$ as $\epsilon \rightarrow 0$.

3.9. If $F(\xi)=P(\xi) / Q(\xi)$ is a rational function satisfying Condition B but not necessarily $B_{1}$ we introduce the functions

$$
F_{\epsilon}(\xi)=P(\xi) /\left(Q(\xi)+\epsilon \frac{\xi^{4}}{1+\xi^{6}}\right) .
$$

By arguments similar to those used in $\$ 3.8$ we can show that for sufficiently small (nonzero) $\epsilon, F_{\epsilon}(\xi)$ will satisfy Condition $B_{1}$, i.e., have only simple poles. Therefore we can apply the result of $\S 3.8$ to the functions $F_{ \pm \epsilon}(\xi)$, with $\epsilon>0$, and, using the Weyl-Courant Lemma, deduce Theorm 3.2 for the function $F(\xi)$.

3.10. To rid ourselves finally of the assumption that $F(\xi)$ is a rational function, we need two simple lemmas. 
Lemma 1. Let $f(x)$ be continuous and bounded in the half-open interval $(0,1]$. Then we can find a bounded sequence of polynomials $\left\{f_{n}(x)\right\}$ such that $f_{n}(x)$ $\geqq f(x)$ in $(0,1]$ for each $n$, and $f_{n}(x) \rightarrow f(x)$ uniformly in every subinterval $[\delta, 1]$.

If $|f(x)| \leqq d$ throughout $(0,1]$, we define

$$
g_{n}(x)= \begin{cases}f(x) & \text { on }\left[\frac{1}{n}, 1\right], \\ f(x)+4 d(1-n x) & \text { on }\left[\frac{1}{2 n}, \frac{1}{n}\right], \\ f\left(\frac{1}{2 n}\right)+2 d & \text { on }\left[0, \frac{1}{2 n}\right] .\end{cases}
$$

Then $g_{n}(x)$ is continuous on $[0,1], g_{n}(x) \geqq f(x)$ in $(0,1]$, and $g_{n}(x)=f(x)$ on $[1 / n, 1]$. If we take as $f_{n}(x)$ a polynomial satisfying

$$
\left|f_{n}(x)-\left(g_{n}(x)+\frac{1}{n}\right)\right|<\frac{1}{2 n} \quad \text { on }[0,1],
$$

the sequence $\left\{f_{n}(x)\right\}$ clearly has the required properties.

Lemma 2. Let $f(x)$ be continuous on $[0,1]$ and $O(x)$ near $x=0$. Then we can find a sequence of polynomials $\left\{f_{n}(x)\right\}$ such that $f_{n}(x) \geqq f(x), f_{n}(0)=0$ for each $n$, and $f_{n}(x) \rightarrow f(x)$ uniformly on $[0,1]$.

This is an immediate consequence of Lemma 1 applied to the function $f(x) / x$.

We can now complete the proofs of the main theorems.

Proof of Theorem 3.1. To approximate $F(\xi)$ by rational functions, we map $(-\infty, \infty)$ into the unit interval and approximate by polynomials. Thus, we set $x=\left(1+\xi^{2}\right)^{-1}$ and define

$$
f(x)=F(\xi)=F\left(\left(x^{-1}-1\right)^{1 / 2}\right) .
$$

Then $f(x)$ is continuous on the unit interval $[0,1]$ and, since $F(\xi)$ is twice continuously differentiable near $\xi=0, f(x)$ is once continuously differentiable near $x=1$. Thus the function

$$
g(x)=\frac{f(x)-M}{1-x}+M
$$

is continuous on $[0,1]$. Moreover since $f(x)=O(x)$ near $x=0$ (here we use the assumption $F(\xi)=O\left(|\xi|^{-2}\right)$ at infinity) we also have $g(x)=O(x)$ near $x=0$. It follows from Lemma 2 that for each $\epsilon>0$ we can find polynomials $g_{ \pm \iota}(x)$ satisfying 


$$
\begin{aligned}
g_{ \pm \epsilon}(0) & =0, \\
g_{-\epsilon}(x) & \leqq g(x) \leqq g_{\epsilon}(x), \\
\left|g(x)-g_{ \pm \epsilon}(x)\right| & \leqq \epsilon .
\end{aligned}
$$

Then if we define

$$
f_{ \pm \epsilon}(x)=M x+(1-x) g_{ \pm \epsilon}(x),
$$

$f_{ \pm \epsilon}(x)$ are polynomials satisfying

$$
\begin{aligned}
f_{ \pm \epsilon}(0) & =0 \\
f_{-\epsilon}(x) & \leqq f(x) \leqq f_{\epsilon}(x), \\
\left|f(x)-f_{ \pm \epsilon}(x)\right| & \leqq \epsilon(1-x) .
\end{aligned}
$$

Finally, defining the rational functions

$$
F_{ \pm \epsilon}(\xi)=f_{ \pm \epsilon}\left(\frac{1}{1+\xi^{2}}\right)
$$

we have

$$
\begin{aligned}
& F_{ \pm \epsilon}(\xi) \in L_{1}(-\infty, \infty), \\
& F_{-\epsilon}(\xi) \leqq F(\xi) \leqq F_{\epsilon}(\xi),
\end{aligned}
$$

$$
\left|F(\xi)-F_{ \pm \epsilon}(\xi)\right| \leqq \epsilon \frac{\xi^{2}}{1+\xi^{2}} .
$$

It follows from (3.22) that for sufficiently small $\epsilon, F_{ \pm \epsilon}(\xi)$ will satisfy Condition $\mathrm{B}$ with $F_{ \pm \epsilon}(0)=M$, and so, using only the first order approximation,

$$
\lambda_{v, A}\left(F_{ \pm \epsilon}\right)=M+\frac{F_{ \pm \epsilon}^{\prime \prime}(0) \pi^{2} v^{2}}{8 A^{2}}+o\left(A^{-2}\right), \quad v=1,2, \cdots
$$

Since, by (3.22), $F_{ \pm \epsilon}^{\prime \prime}(0) \rightarrow-\sigma^{2}$ as $\epsilon \rightarrow 0$, we may apply the Weyl-Courant Lemma to obtain

$$
\lambda_{v, A}(F)=M-\frac{\sigma^{2} \pi^{2} v^{2}}{8 A^{2}}+o\left(A^{-2}\right), \quad v=1,2, \cdots .
$$

Proof of Theorem 3.2. We begin as in the proof of Theorem 3.1 except that we define $g(x)$ not by (3.20) but by

$$
g(x)=\frac{f(x)-M+\sigma^{2}(1-x) / 2}{(1-x)^{2}}+M-\frac{1}{2} \sigma^{2} .
$$

Since $F(\xi)$ is four times continuously differentiable near $\xi=0, f(x)$ is twice continuously differentiable near $x=1$, so $g(x)$ is continuous throughout 
$[0,1]$. Again, since $f(x)=O(x)$ near $x=0, g(x)=O(x)$ near $x=0$, so we can apply Lemma 2 to find polynomials $g_{ \pm \epsilon}(x)$ satisfying the three conditions of (3.21), where now $g(x)$ is defined by (3.23). Setting

$$
f_{ \pm \epsilon}(x)=M-\frac{\sigma^{2}}{2}(1-x)-\left(M-\frac{1}{2} \sigma^{2}\right)(1-x)^{2}+(1-x)^{2} g_{ \pm \epsilon}(x)
$$

we have

$$
\begin{aligned}
f_{ \pm \epsilon}(0) & =0 \\
f_{-\epsilon}(x) \leqq f(x) & \leqq f_{\epsilon}(x), \\
\left|f(x)-f_{ \pm \epsilon}(x)\right| & \leqq \epsilon(1-x)^{2} .
\end{aligned}
$$

Finally, defining the rational functions

$$
F_{ \pm \epsilon}(\xi)=f_{ \pm \epsilon}\left(\frac{1}{1+\xi^{2}}\right)
$$

we have

$$
\begin{aligned}
F_{ \pm \epsilon}(\xi) & \in L_{1}(-\infty, \infty) \\
F_{-\epsilon}(\xi) \leqq F(\xi) & \leqq F_{\epsilon}(\xi), \\
\left|F(\xi)-F_{ \pm \epsilon}(\xi)\right| & \leqq \epsilon \xi^{4} /\left(1+\xi^{2}\right)^{2} .
\end{aligned}
$$

It follows from (3.24) that for sufficiently small $\epsilon, F_{ \pm \epsilon}(\xi)$ will satisfy Condition B with $F_{ \pm \epsilon}(0)=M$ and $F_{ \pm \epsilon}^{\prime \prime}(0)=-\sigma^{2}$ so by $\S 3.9$

$$
\lambda_{v, A}\left(F_{ \pm \epsilon}\right)=M-\frac{\sigma^{2} \pi^{2} v^{2}}{8 A^{2}}\left[1+\frac{\alpha\left(F_{ \pm \epsilon}\right)}{A}\right]+o\left(A^{-3}\right), \quad v=1,2, \cdots .
$$

Now from (3.24) it follows, by an argument entirely analogous to that at the end of $\S 3.8$, that $\alpha\left(F_{ \pm \epsilon}\right) \rightarrow \alpha(F)$ as $\epsilon \rightarrow 0$. Thus we may apply the WeylCourant lemma to obtain Theorem 3.2 for $F(\xi)$.

\section{BIBLIOGRAPHY}

1. R. Bellman and R. Latter, On the integral equation $\lambda f(x)=\int_{0}^{a} K(x-y) f(y) d y$, Proc. Amer. Math. Soc. vol. 3 (1952) pp. 884-891.

2. G. F. Carrier, On the asymptotic integration of integral equations, unpublished report to the Los Alamos Scientific Laboratory.

3. - Integral equation boundary layer problems, 50 Jahre Grenzschichtforschung, Festschrift (for Prandtl), 1954.

4. R. Courant and D. Hilbert, Methoden der mathematischen Physik, vol. 1, Berlin, 1931.

5. M. Kac, W. L. Murdock, and G. Szegö, On the eigenvalues of certain hermitian forms, Journal of Rational Mechanics and Analysis vol. 2 (1953) pp. 767-800.

6. S. Kaczmarz and H. Steinhaus, Theorie der Orthogonalreihen, Warszawa-Lwow, 1935.

Cornell University, ITHACA, N. Y. 